\title{
Conjugacy classes in parabolic subgroups of general linear groups
}

\author{
Anton Evseev* \\ with an appendix by \\ Anton Evseev and George Wellen ${ }^{\dagger}$
}

\begin{abstract}
We prove a formula connecting the number of unipotent conjugacy classes in a maximal parabolic subgroup of a finite general linear group with the numbers of unipotent conjugacy classes in various parabolic subgroups in smaller dimensions. We generalise this formula and deduce a number of corollaries; in particular, we express the number of conjugacy classes of unitriangular matrices over a finite field in terms of the numbers of unipotent conjugacy classes in maximal parabolic subgroups over the same field. We show how the numbers of unipotent conjugacy classes in parabolic subgroups of small dimensions may be calculated.
\end{abstract}

\section{Introduction}

Let $q$ be a prime power and $\mathbb{F}_{q}$ be the finite field with $q$ elements. If $k$ and $m$ are nonnegative integers, let $\mathrm{M}_{m, k}(q)$ be the set of all $m \times k$ matrices over $\mathbb{F}_{q}$. Let $\mathbf{l}=\left(l_{1}, \ldots, l_{s}\right)$ be a sequence of nonnegative integers. Writing $\mathrm{M}_{k}(q)=\mathrm{M}_{k, k}(q)$, let $M^{\mathrm{l}}(q)$ be the set of all matrices of the form

$$
\left(\begin{array}{cccc}
\mathrm{M}_{l_{1}}(q) & \mathrm{M}_{l_{1}, l_{2}}(q) & \ldots & \mathrm{M}_{l_{1}, l_{s}}(q) \\
0 & \mathrm{M}_{l_{2}}(q) & \ldots & \mathrm{M}_{l_{2}, l_{s}}(q) \\
\vdots & \ddots & \ddots & \vdots \\
0 & 0 & \ldots & \mathrm{M}_{l_{s}}(q)
\end{array}\right)
$$

Let $P^{\mathrm{l}}(q)$ be the group of all invertible matrices in $\mathrm{M}^{\mathrm{l}}(q)$. The group $P^{\mathrm{l}}(q)$ is called a parabolic subgroup of the group $\mathrm{GL}_{m}(q)$, where $m=l_{1}+\cdots+l_{s}$. Alternatively, a parabolic subgroup may be described as the stabiliser of a flag in the vector space $\mathbb{F}_{q}^{m}$. Let $N^{\mathbf{l}}(q)$ be the set of all nilpotent matrices in $M^{\mathbf{l}}(q)$.

The group $P^{\mathrm{l}}(q)$ acts on the set $M^{\mathrm{l}}(q)$ by conjugation: ${ }^{g} x=g x g^{-1}(g \in$ $\left.P^{\mathrm{l}}(q), x \in M^{\mathrm{l}}(q)\right)$. We investigate the number of orbits of this action and also the numbers of orbits of the actions of $P^{\mathbf{l}}(q)$ on certain subsets of $M^{1}(q)$.

Throughout the paper, we denote by $\gamma(G, X)$ the number of orbits of an action of a group $G$ on a finite set $X$, where the action is understood. Let

$$
\rho_{\mathbf{l}}(q)=\gamma\left(P^{\mathbf{l}}(q), N^{\mathbf{l}}(q)\right) .
$$

\footnotetext{
*Selwyn College, Cambridge, CB3 9DQ, UK, A.Evseev@dpmms.cam.ac.uk

†Mathematical Institute, 24-29 St Giles', Oxford, OX1 3LB, UK, wellen@maths.ox.ac.uk
} 
Although the groups $P^{\mathrm{l}}(q)$ have received a lot of attention, not much is known about the numbers $\gamma\left(P^{\mathrm{l}}(q), P^{\mathrm{l}}(q)\right)$ and $\rho_{\mathbf{l}}(q)$. In particular, it is not known whether the following is true.

Conjecture 1. For every tuple $\mathbf{l}=\left(l_{1}, \ldots, l_{s}\right)$ of nonnegative integers, $\rho_{\mathbf{l}}(q)$ is polynomial in $q$.

(Here, and in what follows, we call a rational-valued function $f(x)$ defined on a set $D$ of integers polynomial if there exists a polynomial $g$ with rational coefficients such that $f(x)=g(x)$ for all $x \in D$.) Write $\left(1^{m}\right)=\underbrace{(1,1, \ldots, 1)}_{m}$. The following two special cases of Conjecture 1 have received considerable attention and will be of particular interest to us.

Conjecture 2. For every positive integer $m, \rho_{\left(1^{m}\right)}(q)$ is a polynomial in $q$ with rational coefficients.

Conjecture 3. For any positive integers $k$ and $m, \rho_{(k, m)}(q)$ is a polynomial in $q$ with rational coefficients.

It is not difficult to show that Conjecture 2 is equivalent to the conjecture that the number of conjugacy classes of the group of upper unitriangular $n \times n$ matrices is a polynomial in $q$ with rational coefficients (see [4, Section 4.2]). This last conjecture has been proved for $n \leq 13$ by J.M. Arregi and A. VeraLópez [8, 9, 10]. Conjecture 3 has been proved for $k \leq 5$ (or, alternatively, for $m \leq 5)$; indeed, S.H. Murray [7] proved that in those cases $\rho_{(k, m)}(q)$ does not depend on $q$.

A partition is a (possibly, empty) non-increasing sequence of positive integers. If $\lambda=\left(\lambda_{1}, \ldots, \lambda_{r}\right)$ is a partition, let $|\lambda|=\lambda_{1}+\cdots+\lambda_{r}$, and write $l(\lambda)=r$. If $k, m \in \mathbb{N}$, let $\mathcal{P}_{k}$ be the set of all partitions $\lambda$ with $|\lambda|=k$, let $\mathcal{P}^{m}$ be the set of partitions $\lambda$ such that $\lambda_{i} \leq m$ for all $i$, and let $\mathcal{P}_{k}^{m}=\mathcal{P}_{k} \cap \mathcal{P}^{m}$. Let $p(k)=\left|\mathcal{P}_{k}\right|$.

Let $m \in \mathbb{N}$, and let $\lambda=\left(\lambda_{1}, \ldots, \lambda_{r}\right)$ be a partition such that $\lambda_{1} \leq m$. Define

$$
\delta(m, \lambda)=\left(\lambda_{r}, \lambda_{r-1}-\lambda_{r}, \lambda_{r-2}-\lambda_{r-1}, \ldots, \lambda_{1}-\lambda_{2}, m-\lambda_{1}\right) .
$$

Let $\nu_{\lambda}^{m}(q)=\rho_{\delta(m, \lambda)}(q)$. Thus, $\nu_{\lambda}^{m}(q)$ is the number of $P^{\mathrm{l}}(q)$-orbits of $N^{\mathrm{l}}(q)$ where $P^{\mathbf{l}}(q)$ is the stabiliser of a flag

$$
\mathbb{F}_{q}^{\lambda_{r}} \leq \mathbb{F}_{q}^{\lambda_{r-1}} \leq \cdots \leq \mathbb{F}_{q}^{\lambda_{1}} \leq \mathbb{F}_{q}^{m}
$$

We shall generalise the definition of $\nu_{\lambda}^{m}(q)$ as follows. Let $\overline{\mathbb{F}}_{q}$ be the algebraic closure of the field $\mathbb{F}_{q}$, and let $F$ be a subset of $\overline{\mathbb{F}}_{q}$. Let $\mathcal{Y}=\mathcal{Y}_{F}$ be the family of all linear endomorphisms $T: U \rightarrow U$ (where $U$ is an arbitrary finite dimensional vector space over $\mathbb{F}_{q}$ ) such that all eigenvalues of $T$ over $\overline{\mathbb{F}}_{q}$ are in $F$. We shall say that $\mathcal{Y}$ is the class of endomorphisms associated with $F$. We shall refer to elements of this class as $\mathcal{Y}$-endomorphisms. Obviously, $\mathcal{Y}$ is preserved by conjugation. Note that the family of all nilpotent endomorphisms and the family of all invertible endomorphisms are both classes. If $\mathcal{Y}$ is a class and $m \in \mathbb{Z}_{\geq 0}$, let $c(m, \mathcal{Y})$ be the number of $\mathrm{GL}_{m}\left(\mathbb{F}_{q}\right)$-orbits on $\mathcal{Y} \cap \mathrm{M}_{m}\left(\mathbb{F}_{q}\right)$. We shall denote by $\mathcal{N}$ the class of all nilpotent endomorphisms. Note that

$$
c(m, \mathcal{N})=p(m)
$$

the number of partitions of $m$. This follows from the fact that nilpotent matrices in Jordan canonical form form a complete set of representatives of $\mathrm{GL}_{m}(q)$ orbits on $\mathrm{N}_{m}(q)$, the set of nilpotent $m \times m$ matrices. 
Let $\mathcal{Y}$ be a class (of endomorphisms), and let $m \in \mathbb{N}$. Let $\lambda=\left(\lambda_{1}, \ldots, \lambda_{r}\right) \in$ $\mathcal{P}^{m}$ (that is, $\lambda_{1} \leq m$ ). Define

$$
\kappa_{\lambda}^{m}(\mathcal{Y})=\gamma\left(P^{\delta(m, \lambda)}(q), M^{\delta(m, \lambda)}(q) \cap \mathcal{Y}\right) .
$$

One of the main results of this paper is as follows.

Theorem 1.1. Let $k$ and $m$ be positive integers. Let $q$ be a prime power and $\mathcal{Y}$ be a class of endomorphisms over $\mathbb{F}_{q}$. Then

$$
\kappa_{(k)}^{k+m}(\mathcal{Y})=\sum_{j=0}^{k} c(k-j, \mathcal{Y}) \sum_{\lambda \in \mathcal{P}_{j}^{m}} \kappa_{\lambda}^{m}(\mathcal{Y}) .
$$

In particular,

$$
\rho_{(k, m)}(q)=\sum_{j=0}^{k} p(k-j) \sum_{\lambda \in \mathcal{P}_{j}^{m}} \nu_{\lambda}^{m}(q) .
$$

Remark. S.H. Murray [6] has proved a similar result stated in terms of irreducible representations of parabolic subgroups. This result implies Theorem 1.1 in the case when $\mathcal{Y}$ is the class of all invertible matrices. The proof in 6 is different from the one given here. Unlike the proof in this paper, the proof in [6] establishes not just a numerical equality, but also an explicit correspondence between representations. It is an interesting question whether the method of [ 6 ] can be extended to prove an analogue of the more general Theorem 6.2.

We will deduce the following two results from Theorem 1.1

Theorem 1.2. Let $m \in \mathbb{N}$, and let $n=m(m-1) / 2$. There exist integers $a_{0}, a_{1}, \ldots, a_{n}$ such that, for all prime powers $q$,

$$
\rho_{\left(1^{m}\right)}(q)=\sum_{j=0}^{n} a_{j} \rho_{(j, m)}(q) .
$$

Hence, Conjecture 3 implies Conjecture 2 .

If $\mathbf{l}=\left(l_{1}, \ldots, l_{s}\right)$, write $\rho_{k, \mathbf{l}}(q)$ for $\rho_{\left(k, l_{1}, \ldots, l_{s}\right)}(q)$.

Theorem 1.3. Let $k, m \in \mathbb{N}$, and let $n=m(m-1) / 2$. There exist integers $a_{k 0}, \ldots, a_{k n}$ such that, for all tuples $\mathbf{l}=\left(l_{1}, \ldots, l_{s}\right)$ of nonnegative integers with $l_{1}+\cdots+l_{s}=m$,

$$
\rho_{k, \mathbf{l}}(q)=\sum_{j=0}^{n} a_{k j} \rho_{j, \mathbf{l}}(q)
$$

Using the methods developed in the proof of Theorem 1.1, one may compute $\rho_{\mathbf{l}}(q)$ for all tuples $\mathbf{l}=\left(l_{1}, \ldots, l_{s}\right)$ with $l_{1}+\cdots+l_{s} \leq 6$. In particular, the following holds.

Proposition 1.4. Let $\mathbf{l}=\left(l_{1}, \ldots, l_{s}\right)$ be a tuple of nonnegative integers with $l_{1}+\cdots+l_{s} \leq 6$. Then $\rho_{\mathbf{l}}(q)$ is a polynomial in $q$ with positive integer coefficients. Hence (by Theorem 1.1) $\rho_{(k, m)}(q)$ is polynomial in $q$ whenever $m \leq 6$ and $k \in \mathbb{N}$.

This paper is organised as follows. In Section 2 we describe a general framework of quiver representations and their endomorphisms, which is used to state and prove the results. In Section 3 we show, in particular, how the numbers $\gamma\left(P^{\mathbf{l}}(q), P^{\mathbf{l}}(q)\right)$ and $\gamma\left(P^{\mathbf{l}}(q), M^{\mathbf{l}}(q)\right)$ may be expressed in terms of $\rho_{\mathbf{1}^{\prime}}\left(q^{d}\right)$ where $d \in \mathbb{N}$ and $\mathbf{l}^{\prime}$ is another tuple of nonnegative integers. This justifies our focus on the numbers $\rho_{\mathbf{l}}(q)$. 
Section 4 contains a few standard results used later. In Section 5 we prove results that serve as the main tools allowing us to reduce problems such as that of counting $\rho_{(k, m)}(q)$ to other problems in a smaller dimension. In Section 6 we use those tools to prove a generalisation of Theorem 1.1 stated in terms of quiver representations (Theorem 6.2).

In Section 7 we invert the formulae in Theorems 1.1 and 6.2 and deduce Theorems 1.2 and 1.3. This relies on combinatorial results proved jointly with G. Wellen in the Appendix. I am very grateful to George Wellen for his part in this work.

In Section 8 we describe a method for computing $\rho_{\mathbf{l}}(q)$ when $m=l_{1}+\cdots+$ $l_{s}$ is small. For this purpose, we generalise our problem to that of counting conjugacy classes of groups associated with preordered sets. Finally, Section 9 investigates the symmetry afforded by considering a dual quiver representation. In particular, we show that

$$
\rho_{\left(l_{1}, \ldots, l_{s}\right)}(q)=\rho_{\left(l_{s}, \ldots, l_{1}\right)}(q) .
$$

Acknowledgments. Most of this paper is a part of my D.Phil. thesis. I am very grateful to my supervisor, Marcus du Sautoy, and to George Wellen for his part in this work. I would also like to thank my thesis examiners, Dan Segal and Gerhard Röhrle, for spotting a number of errors and for helpful comments.

\section{Notation and definitions}

- $\gamma(G, X)$ is the number of $G$-orbits on a finite set $X$ where the action of a group $G$ on $X$ is understood;

- $[k, n]=\{k, k+1, k+2, \ldots, n\}$ where $k \leq n$ are integers;

- $|X|$ is the cardinality of a set $X$;

- $\mathcal{A}$ is a partition of a set $X$ if $\mathcal{A}$ is a family of disjoint sets whose union is $X$; two elements $x$ and $y$ of $X$ are said to be $\mathcal{A}$-equivalent if there exists $A \in \mathcal{A}$ such that $x, y \in A$;

- $\delta_{i j}=0$ if $i \neq j$, and $\delta_{i i}=1$;

- $A \sqcup B$ is the disjoint union of sets $A$ and $B$ (formally defined as $A \times\{0\} \cup$ $B \times\{1\})$;

- $I_{V}=I$ is the identity element of $\mathrm{GL}(V)$;

- $I_{k}$ is the identity $k \times k$ matrix over an appropriate field.

- Suppose $U$ and $V$ are vector spaces, $X \in \operatorname{End}(U)$ and $Y \in \operatorname{End}(V)$; then $\mathcal{I}(X, Y)$ denotes the vector space of all linear maps $T: U \rightarrow V$ such that $T X=Y T$

- $\mathrm{N}_{n}(K)$ is the set of all nilpotent matrices in $\mathrm{M}_{n, n}(K)$;

- $\operatorname{End}\left(V ; U_{1}, \ldots, U_{k}\right)=\left\{f \in \operatorname{End}(V): f\left(U_{i}\right) \subseteq U_{i} \forall i\right\}$ where $U_{i}$ are subspaces of $V$;

- $\mathscr{P}\left(V ; U_{1}, \ldots, U_{k}\right):=\operatorname{End}\left(V ; U_{1}, \ldots, U_{k}\right) \cap \mathrm{GL}(V)$;

- By convention, the set of $0 \times k$ matrices contains just one element (which is nilpotent if $k=0$ ), and the group $\mathrm{GL}_{0}(K)$ is trivial;

- If $I$ and $J$ are finite sets, then $\mathrm{M}_{I, J}(K)$ is the set of all matrices over a field $K$ whose rows are indexed by the elements of $I$ and whose columns are indexed by elements of $J$; we refer to these as $I \times J$ matrices; note that, if $A \in \mathrm{M}_{I, J}(K)$ and $B \in \mathrm{M}_{J, J^{\prime}}(K)$, then the product $A B \in \mathrm{M}_{I, J^{\prime}}(K)$ is well defined; 
- $A^{t}$ is the transpose of a matrix $A$;

- $\operatorname{Tr}(A)$ is the trace of a square matrix $A$;

- If a group $G$ acts on a set $X$, two elements of $X$ are said to be $G$-conjugate if they are in the same $G$-orbit;

- All rings are understood to have an identity element;

- If $R$ is a ring, then $R^{\text {op }}$ is the ring with the same underlying abelian group and with the multiplication $(r, s) \mapsto s r$;

\section{Quiver representations and automorphisms}

We recall the standard definitions related to quivers (see [1], for example). A quiver is a pair $\left(E_{0}, E_{1}\right)$ of finite sets together with maps $\sigma: E_{1} \rightarrow E_{0}$ and $\tau: E_{1} \rightarrow E_{0}$. Elements of $E_{0}$ may be thought of as nodes; then each element $e \in E_{1}$ may be represented as an arrow from $\sigma(e)$ to $\tau(e)$. Let $K$ be a field. A representation of a quiver $\left(E_{0}, E_{1}\right)$ over $K$ is a pair $(\mathbf{U}, \boldsymbol{\alpha})$ such that

(i) $\mathbf{U}=\left(U_{a}\right)_{a \in E_{0}}$ is a tuple of vector spaces over $K$;

(ii) $\boldsymbol{\alpha}=\left(\alpha_{e}\right)_{e \in E_{1}}$ where $\alpha_{e} \in \operatorname{Hom}\left(U_{\sigma(e)}, U_{\tau(e)}\right)$.

If $(\mathbf{U}, \boldsymbol{\alpha})$ is a representation of a quiver $\left(E_{0}, E_{1}\right)$, we shall refer to the quadruple $Q=\left(E_{0}, E_{1}, \mathbf{U}, \boldsymbol{\alpha}\right)$ as a quiver representation. A quiver representation may be thought of as a collection of vector spaces together with linear maps between some of those spaces.

If $(\mathbf{U}, \boldsymbol{\alpha})$ and $\left(\mathbf{U}^{\prime}, \boldsymbol{\alpha}^{\prime}\right)$ are two representations of a quiver $\left(E_{0}, E_{1}\right)$, a morphism between those representations is a tuple $\left(X_{a}\right)_{a \in E_{0}}$ such that

(i) $X_{a} \in \operatorname{Hom}\left(U_{a}, U_{a}^{\prime}\right)$ for all $a \in E_{0}$;

(ii) $\alpha_{e}^{\prime} X_{\sigma(e)}=X_{\tau(e)} \alpha_{e}$ for all $e \in E_{1}$.

This defines the category of representations of $\left(E_{0}, E_{1}\right)$.

If $Q=\left(E_{0}, E_{1}, \mathbf{U}, \boldsymbol{\alpha}\right)$ is a quiver representation, write $\operatorname{End}(Q)$ for the ring of all endomorphisms of $Q$ and $\operatorname{Aut}(Q)$ for the group of all automorphisms of $Q$. Let $N(Q)$ be the set of all nilpotent elements of $\operatorname{End}(Q)$. The group $\operatorname{Aut}(Q)$ acts naturally on the set $\operatorname{End}(Q)$ by conjugation: if $\mathbf{X}=\left(X_{a}\right) \in \operatorname{End}(Q)$ and $\mathrm{g} \in \operatorname{Aut}(Q)$, then

$$
\mathbf{g} \circ \mathbf{X}=\left(g_{a} X_{a} g_{a}^{-1}\right)_{a \in E_{0}} .
$$

Assume that $K=\mathbb{F}_{q}$, where $q$ is a prime power. We shall be concerned with the number of orbits of this action and, more generally, with the number of orbits of actions of certain subgroups of $\operatorname{Aut}(Q)$ on certain subsets of $\operatorname{End}(Q)$, such as $N(Q)$ for example. Note that $\operatorname{Aut}(Q)$ and $N(Q)$ may be defined in terms of the ring structure on $\operatorname{End}(Q)$, so they are preserved by ring isomorphisms. Let

$$
\theta(Q)=\gamma(\operatorname{Aut}(Q), N(Q))
$$

All the problems discussed in the introduction may be stated in terms of quiver representations. If $\mathbf{l}=\left(l_{1}, l_{2}, \ldots, l_{s}\right)$ is a tuple of nonnegative integers with $m=l_{1}+\cdots+l_{s}$, let $R_{\mathbf{l}}=R_{\mathbf{l}}(q)$ be the quiver representation

$$
\mathbb{F}_{q}^{l_{1}} \longleftrightarrow \mathbb{F}_{q}^{l_{1}+l_{2}} \leftrightharpoons \cdots \smile \mathbb{F}_{q}^{l_{1}+\cdots+l_{s-1}} \bigcup^{\longrightarrow} \mathbb{F}_{q}^{m}
$$

where all the arrows represent injective linear maps. More formally,

$$
R_{\mathbf{l}}=([1, s],[1, s-1], \mathbf{U}, \boldsymbol{\alpha})
$$


with $\sigma(i)=i, \tau(i)=i+1$ for all $i \in[1, s-1], U_{i}=\mathbb{F}_{q}^{l_{1}+\cdots+l_{i}}$ and $\alpha_{i}$ injective. Obviously, these conditions define $R_{1}$ up to isomorphism of representations.

We may choose a basis $\left\{b_{1}, \ldots, b_{m}\right\}$ of $U_{s}=\mathbb{F}_{q}^{m}$ so that, for each $i$, the image of $U_{i}=\mathbb{F}_{q}^{l_{1}+\cdots+l_{i}}$ under $\alpha_{s-1} \cdots \alpha_{i+1} \alpha_{i}$ is equal to the span of $\left\{b_{1}, \ldots, b_{l_{1}+\cdots+l_{i}}\right\}$. Let $J_{\mathbf{l}}: \operatorname{End}\left(R_{\mathbf{l}}\right) \rightarrow \mathrm{M}_{m, m}(q)$ be the map which assigns to each $\mathbf{X}=\left(X_{i}\right)_{i \in[1, s]} \in$ $\operatorname{End}\left(R_{\mathbf{l}}\right)$ the matrix of $X_{s} \in \operatorname{End}\left(\mathbb{F}_{q}^{m}\right)$ with respect to the basis $\left\{b_{1}, \ldots, b_{m}\right\}$. Then the following result is obvious.

Lemma 2.1. The map $J_{\mathbf{l}}$ is a ring isomorphism from $\operatorname{End}\left(R_{\mathbf{l}}\right)$ onto $M^{\mathbf{l}}(q)$. Hence, $\rho_{\mathbf{l}}(q)=\theta\left(R_{\mathbf{l}}\right)$.

Call a quiver representation $Q^{\prime}=\left(E_{0}^{\prime}, E_{1}^{\prime}, \mathbf{U}^{\prime}, \boldsymbol{\alpha}^{\prime}\right)$ an extension of a quiver representation $Q=\left(E_{0}, E_{1}, \mathbf{U}, \boldsymbol{\alpha}\right)$ if

(i) $E_{0}^{\prime} \supseteq E_{0}$;

(ii) $U_{a}^{\prime}=U_{a}$ for all $a \in E_{0}$;

(iii) for every $\mathbf{X}=\left(X_{a}\right)_{a \in E_{0}^{\prime}} \in \operatorname{End}\left(Q^{\prime}\right)$, the tuple $\left(X_{a}\right)_{a \in E_{0}}$ belongs to $\operatorname{End}(Q)$. by

Let $Q^{\prime}$ be an extension of $Q$. Define a map $\pi=\pi_{Q}^{Q^{\prime}}: \operatorname{End}\left(Q^{\prime}\right) \rightarrow \operatorname{End}(Q)$

$$
\left(X_{a}\right)_{a \in E_{0}^{\prime}} \mapsto\left(X_{a}\right)_{a \in E_{0}}
$$

If $B \subseteq \operatorname{End}(Q)$, let $B^{Q^{\prime}}=\pi^{-1}(B)$. If $G$ is a subgroup of $\operatorname{Aut}(Q)$, define $G^{Q^{\prime}}=\pi^{-1}(G) \cap \operatorname{Aut}\left(Q^{\prime}\right)$. (This will cause no ambiguity if a group is considered a distinct object from the set of its elements.)

Let $Q=\left(E_{0}, E_{1}, \mathbf{U}, \boldsymbol{\alpha}\right)$ be a quiver representation, and let $e \in E$ with $\sigma(e)=a, \tau(e)=b$. Let $Y=\operatorname{ker}\left(\alpha_{e}\right) \leq U_{a}, Z=\operatorname{im}\left(\alpha_{e}\right) \leq U_{b}$. Define an extension $K(Q, e)=\left(E_{0}^{\prime}, E_{1}^{\prime}, \mathbf{U}^{\prime}, \boldsymbol{\alpha}^{\prime}\right)$ as follows:

(i) $E_{0}^{\prime}=E_{0} \sqcup\{c\}$;

(ii) $U_{c}^{\prime}=Y\left(\right.$ and $U_{x}^{\prime}=U_{x}$ for $\left.x \in E_{0}\right)$;

(iii) $E_{1}^{\prime}=E_{1} \sqcup\left\{e^{\prime}\right\}$ where $\sigma\left(e^{\prime}\right)=c, \tau\left(e^{\prime}\right)=a$;

(iv) $\alpha_{f}^{\prime}=\alpha_{f}$ for all $f \in E_{1}$, and $\alpha_{e^{\prime}}^{\prime}$ is the inclusion map $Y \hookrightarrow U_{a}$.

Define another extension $I(Q, e)=\left(E_{0}^{\prime \prime}, E_{1}^{\prime \prime}, \mathbf{U}^{\prime \prime}, \boldsymbol{\alpha}^{\prime \prime}\right)$ as follows:

(i) $E_{0}^{\prime \prime}=E_{0} \sqcup\{d\}$;

(ii) $U_{d}^{\prime \prime}=Z$;

(iii) $E_{1}^{\prime \prime}=\left(E_{1} \backslash\{e\}\right) \sqcup\left\{e^{\sharp}, e^{\prime \prime}\right\}$, where $\sigma\left(e^{\prime \prime}\right)=d, \tau\left(e^{\prime \prime}\right)=b, \sigma\left(e^{\sharp}\right)=a$, $\tau\left(e^{\sharp}\right)=d ;$

(iv) $\alpha_{f}^{\prime \prime}=\alpha_{f}$ for all $f \in E_{1} \backslash\{e\}, \alpha_{e^{\prime \prime}}^{\prime \prime}$ is the inclusion map $Z \hookrightarrow U_{b}$, and $\alpha_{e^{\sharp}}^{\prime \prime}$ is the map $U_{a} \rightarrow Z$ given by $\alpha_{e^{\sharp}}^{\prime \prime}(v)=\alpha_{e}(v) \forall v \in V$.

Clearly, $K(Q, e)$ is an extension of $Q$. Also, $Q^{\prime \prime}:=I(Q, e)$ is an extension of $Q$ : if $\mathbf{X} \in \operatorname{End}\left(Q^{\prime \prime}\right)$, then $\alpha_{e} X_{a}=X_{b} \alpha_{e}$ because $\alpha_{e}=\alpha_{e^{\prime \prime}}^{\prime \prime} \alpha_{e^{\sharp}}^{\prime \prime}$.

Lemma 2.2. Let $Q=\left(E_{0}, E_{1}, \mathbf{U}, \boldsymbol{\alpha}\right)$ be a quiver representation, and let $e \in E_{1}$. Let $Q^{\prime}=K(Q, e)$ and $Q^{\prime \prime}=I(Q, e)$. Then the maps $\pi_{Q}^{Q^{\prime}}: \operatorname{End}\left(Q^{\prime}\right) \rightarrow \operatorname{End}(Q)$ and $\pi_{Q}^{Q^{\prime \prime}}: \operatorname{End}\left(Q^{\prime \prime}\right) \rightarrow \operatorname{End}(Q)$ are ring isomorphisms.

Proof. As above, let $a=\sigma(e), b=\tau(e), Y=\operatorname{ker}\left(\alpha_{e}\right), Z=\operatorname{im}\left(\alpha_{e}\right)$. Let $\mathbf{X}=\left(X_{i}\right)_{i \in E_{0}} \in \operatorname{End}(Q)$. Since $\alpha_{e} X_{a}=X_{b} \alpha_{e}$, the map $X_{a}$ preserves $Y$ and $X_{b}$ preserves $Z$. Any element $\mathbf{X}^{\prime} \in\left(\pi_{Q}^{Q^{\prime}}\right)^{-1}(\mathbf{X})$ satisfies $\alpha_{e^{\prime}}^{\prime} X_{c}^{\prime}=X_{c}^{\prime} \alpha_{e^{\prime}}^{\prime}$. 
Since $\alpha_{e^{\prime}}^{\prime}$ is injective, it follows that $\left(\pi_{Q}^{Q^{\prime}}\right)^{-1}(\mathbf{X})=\left\{\mathbf{X}^{\prime}\right\}$, where $X_{c}^{\prime}=\left.X_{a}\right|_{Y}$. Similarly, $\left(\pi_{Q}^{Q^{\prime \prime}}\right)^{-1}(\mathbf{X})=\left\{\mathbf{X}^{\prime \prime}\right\}$, where $X_{d}^{\prime \prime}=\left.X_{b}\right|_{Z}$. Hence, $\pi_{Q}^{Q^{\prime}}$ and $\pi_{Q}^{Q^{\prime \prime}}$ are bijections.

\section{Reduction to nilpotent endomorphisms}

In this section we show that, if $\mathcal{Y}$ is a class of endomorphisms over $\mathbb{F}_{q}$, the problem of counting orbits of $\mathcal{Y}$-endomorphisms of a quiver representation may be reduced to that of counting orbits of nilpotent endomorphisms of various quiver representations. (A $\mathcal{Y}$-endomorphism is an endomorphism $\mathbf{X}$ such that $X_{a} \in \mathcal{Y}$ for all $a$.) We use standard methods related to rational canonical forms. The results of this section are not used elsewhere in the paper, but provide some motivation for our later focus on nilpotent endomorphisms.

Let $U$ be a vector space over a field $K$. Let $L$ be a field containing $K$. Suppose $U^{\prime}$ is a vector space over $L$ that becomes $U$ if one restricts the scalars to $K$; that is, $U^{\prime}=U$ as sets and multiplication by scalars from $K$ in $U$ is the same as in $U^{\prime}$. We shall say that $U^{\prime}$ is an $L$-expansion of $U$ and $U$ is the restriction of $U^{\prime}$ to $K$. Let $Q=\left(E_{0}, E_{1}, \mathbf{U}, \boldsymbol{\alpha}\right)$ be a quiver representation over $K$. Say that a quiver representation $Q^{\prime}=\left(E_{0}, E_{1}, \mathbf{U}^{\prime}, \boldsymbol{\alpha}\right)$ over $L$ is an $L$-expansion of $Q$ (and $Q$ is the restriction of $Q^{\prime}$ to $K$ ) if, for each $a \in E_{0}$, the space $U_{a}^{\prime}$ is an $L$-expansion of $U_{a}$. (Then $\alpha_{e}$ is $L$-linear for each $e \in E_{1}$.)

Let $\mathbf{X} \in \operatorname{End}(Q)$. If $a \in E_{0}$ and $f$ is a monic irreducible polynomial over $\mathbb{F}_{q}$, let

$$
U_{\mathbf{X}, f, a}=\left\{u \in U_{a}: f\left(X_{a}\right)^{k} u=0 \text { for some } k \in \mathbb{N}\right\} .
$$

Then $U_{\mathbf{X}, f, a}=0$ for all but finitely many $f$. For all monic irreducible polynomials $f \in \mathbb{F}_{q}[T]$ and all $e \in E_{1}$, we have $\alpha_{e}\left(U_{\mathbf{X}, f, \sigma(e)}\right) \leq U_{\mathbf{X}, f, \tau(e)}$. Thus, $\mathbf{U}_{\mathbf{X}, f}:=\left(U_{X, f, a}\right)_{a \in E_{0}}$ induces a subrepresentation $Q \mathbf{X}, f$ of $Q$.

Lemma 3.1. Let $Q$ be a quiver representation over $\mathbb{F}_{q}$. Suppose $\mathbf{X} \in \operatorname{End}(Q)$. Then

$$
Q=\bigoplus_{f} Q_{\mathbf{X}, f}
$$

where the sum is over all monic irreducible polynomials $f$ over $\mathbb{F}_{q}$. Moreover, the isomorphism class of each component $Q_{\mathbf{X}, f}$ is an invariant of the $\operatorname{Aut}(Q)$ orbit of $\mathbf{X}$.

Proof. The first statement follows from the fact that $U_{a}=\bigoplus_{f} U_{\mathbf{X}, f, a}$ for each $a$. The second statement is clear.

We now consider each quiver representation $Q_{\mathbf{X}, f}$ separately. Fix a monic irreducible polynomial $f=f(T)$ over $\mathbb{F}_{q}$. Let $d$ be the degree of $f$. Suppose $\mathbf{X}$ is an endomorphism of a quiver representation $Q$ over $\mathbb{F}_{q}$ such that $Q=Q \mathbf{X}, f$. Let $\mathbb{F}_{q}[T]_{(f)}$ be the localisation of $\mathbb{F}_{q}[T]$ at the ideal $(f)$; it consists of all fractions $g / h$ such that $g, h \in \mathbb{F}_{q}[T]$ and $h$ is not divisible by $f$. Then $\mathbf{X}$ induces an $\mathbb{F}_{q}[T]_{(f)}$-module structure on each $U_{a}$ : multiplication by $T$ is given by the action of $X_{a}$. Moreover, each $\alpha_{e}$ becomes an $\mathbb{F}_{q}[T]_{(f)}$-module homomorphism.

Let $S_{f}$ be the completion of the discrete valuation ring $\mathbb{F}_{q}[T]_{(f)}$. That is, $S_{f}$ is the inverse limit of the rings $\mathbb{F}_{q}[T] /\left(f^{k}\right), k=1,2, \ldots$. Any finite $\mathbb{F}_{q}[T]_{(f)^{-}}$ module is annihilated by $f^{k}$ for large enough $k$. Thus, a finite $\mathbb{F}_{q}[T]_{(f)}$-module may be thought of as a (finite) $S_{f}$-module (and vice versa). 
Now, by [2, $\S 9$, Proposition 3], $S_{f}$ is isomorphic to $\mathbb{F}_{q^{d}}[[Z]]$, the ring of formal power series over a variable $Z$. Indeed, let $r$ be the element of $S_{f}$ whose projection onto $\mathbb{F}_{q}[T] /\left(f^{k}\right)$ is $T^{q^{k d}}$ for each $k \in \mathbb{N}$. Then $\mathbb{F}_{q}[r] \subseteq S_{f}$ is a field isomorphic to $\mathbb{F}_{q^{d}}$, and each element of $S_{f}$ may be represented as a power series in $f$ with coefficients in $\mathbb{F}_{q}[r]$ (see loc. cit. for more detail).

Hence, $Q$ gives rise to a finite $\mathbb{F}_{q^{d}}[[Z]]$-module. This module corresponds

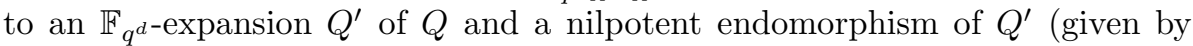
multiplication by $Z$ ).

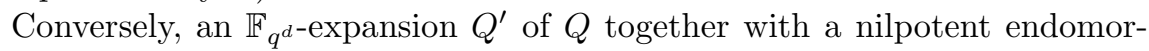
phism of $Q^{\prime}$ gives rise to an $\mathbb{F}_{q^{d}}[[Z]]$-module structure on each $U_{a}$ in such a way that all $\alpha_{e}$ are $\mathbb{F}_{q^{d}}[[Z]]$-endomorphisms. Identifying $\mathbb{F}_{q^{d}}[[Z]]$ with $S_{f}$ as above, we get an endomorphism $\mathbf{X}$ of $Q$ such that $Q_{\mathbf{X}, f}=Q$. We have proved the following result.

Lemma 3.2. Let $q$ be a prime power, and let $f$ be a monic irreducible polynomial over $\mathbb{F}_{q}$ of degree d. Suppose $Q$ is a quiver representation over $\mathbb{F}_{q}$. Then the $\operatorname{Aut}(Q)$-orbits of endomorphisms $\mathbf{X}$ of $Q$ such that $Q_{\mathbf{X}, f}=Q$ are in a oneto-one correspondence with $\operatorname{Aut}(Q)$-orbits of pairs $\left(Q^{\prime}, \mathbf{Z}\right)$ such that $Q^{\prime}$ is an $\mathbb{F}_{q^{d}}$-expansion of $Q$ and $\mathbf{Z} \in N\left(Q^{\prime}\right)$.

Let $\mathcal{Y}$ be a class of linear endomorphisms over $\mathbb{F}_{q}$, as defined in Section 1 Let

$$
\operatorname{End}_{\mathcal{Y}}(Q)=\left\{\mathbf{X} \in \operatorname{End}(Q): X_{a} \in \mathcal{Y} \text { for all } a \in E_{0}\right\} .
$$

Let $F$ be a subset of $\overline{\mathbb{F}}_{q}$ such that $\mathcal{Y}=\mathcal{Y}_{F}$. Call a polynomial $f \in \mathbb{F}_{q}[T]$ a $\mathcal{Y}$-polynomial if all the roots of $f$ (over $\overline{\mathbb{F}}_{q}$ ) are in $F$. Let $k_{\mathcal{Y}, d}(q)$ be the number of monic irreducible $\mathcal{Y}$-polynomials of degree $d$.

Consider again an arbitrary quiver representation $Q=\left(E_{0}, E_{1}, \mathbf{U}, \boldsymbol{\alpha}\right)$ over $\mathbb{F}_{q}$. Lemmata 3.1 and 3.2 allow us to express $\gamma\left(\operatorname{Aut}(Q), \operatorname{End}_{\mathcal{Y}}(Q)\right)$ in terms of $\theta\left(Q_{0}^{\prime}\right)$ where $Q_{0}$ varies among direct summands of $Q$ and $Q_{0}^{\prime}$ is an expansion of $Q_{0}$.

Let $\mathbf{X} \in \operatorname{End}_{\mathcal{Y}}(Q)$. By Lemma 3.1 there exists a finite set $\left\{f_{i}\right\}_{i \in J}$ of irreducible $\mathcal{Y}$-polynomials such that $Q=\bigoplus_{j \in J} Q_{j}$ where $Q_{j}=Q_{\mathbf{X}, f_{j}}$. Here, $J$ is a finite indexing set. Let $\epsilon(j)=\operatorname{deg} f_{j}$. By Lemma 3.2. each $Q_{j}$ together with the restriction of $\mathbf{X}$ to $Q_{j}$ corresponds to an $\mathbb{F}_{q^{\epsilon(j)}}$-expansion $Q_{j}^{\prime}$ of $Q_{j}$ together with a nilpotent endomorphism $\mathbf{Z}^{(j)} \in N\left(Q_{j}^{\prime}\right)$. Up to conjugation by $\operatorname{Aut}\left(Q_{j}^{\prime}\right)$, the endomorphism $\mathbf{Z}^{(j)}$ may be chosen in $\theta\left(Q_{j}^{\prime}\right)$ ways (by definition). Let $\mathcal{A}$ be the partition of $J$ that consists of the sets

$$
\left\{j \in J: Q_{j}^{\prime} \simeq Q_{i}^{\prime}\right\}
$$

where $i$ varies among the elements of $J$. In particular, $\epsilon(i)=\epsilon(j)$ whenever $i$ and $j$ are $\mathcal{A}$-equivalent.

Proposition 3.3. Let $Q$ be a quiver representation over $\mathbb{F}_{q}$. Then

$$
\begin{aligned}
\gamma\left(\operatorname{Aut}(Q), \operatorname{End}_{\mathcal{Y}}(Q)\right) & =\sum_{\left(Q_{i}\right)_{i \in J}} \sum_{\mathcal{A}} \frac{1}{\prod_{A \in \mathcal{A}}|A| !} \sum_{\epsilon} \prod_{d=1}^{\infty} \frac{k \mathcal{Y}, d(q) !}{\left(k_{\mathcal{Y}, d}(q)-\left|\epsilon^{-1}(d)\right|\right) !} \\
& \times \sum_{\left(Q_{A}^{\prime}\right)_{A \in \mathcal{A}}} \prod_{A \in \mathcal{A}} \theta\left(Q_{A}^{\prime}\right)^{|A|}
\end{aligned}
$$

Here, the first sum is over all decompositions $Q=\bigoplus_{i \in J} Q_{i}$ of $Q$ as a direct sum of representations; two such decompositions are considered to be the same if one may be obtained from the other by permuting the indices $i$ and replacing 
each $Q_{i}$ with an isomorphic representation (we assume that $J=[1,|J|]$ ). The second sum is over all partitions $\mathcal{A}$ of $J$ such that $Q_{i} \simeq Q_{j}$ whenever $i$ and $j$ are $\mathcal{A}$-equivalent. The third sum is over all maps $\epsilon: J \rightarrow \mathbb{N}$ such that $\epsilon(i)=\epsilon(j)$ whenever $i$ and $j$ are $\mathcal{A}$-equivalent. The last sum is over all isomorphism classes of tuples $\left(Q_{A}^{\prime}\right)_{A \in \mathcal{A}}$ where $Q_{A}^{\prime}$ is an $\mathbb{F}_{q^{\epsilon(i)}}$-expansion of $Q_{i}$ ( $i$ is an arbitrary element of $A)$ and the quiver representations $Q_{A}^{\prime}(A \in \mathcal{A})$ are pairwise not isomorphic.

Proof. Suppose $\mathcal{A}, \epsilon, Q_{A}^{\prime}=Q_{i}^{\prime}(i \in A)$, as above, are fixed. There are

$$
\prod_{d=1}^{\infty} \frac{k_{\mathcal{Y}, d}(q) !}{\left(k_{\mathcal{Y}, d}(q)-\left|\epsilon^{-1}(d)\right|\right) !}
$$

ways to assign a monic irreducible $\mathcal{Y}$-polynomial $f_{i}$ of degree $\epsilon(i)$ to each $i \in J$ so that all those polynomials are distinct. (Note that all but finitely many terms in the product are equal to 1 , so the product is well defined.) There are

$$
\prod_{A \in \mathcal{A}} \theta\left(Q_{A}^{\prime}\right)^{|A|}
$$

ways to choose, for each $i \in J$, an $\operatorname{Aut}\left(Q_{i}^{\prime}\right)$-orbit in $N\left(Q_{i}^{\prime}\right)$. By Lemmata 3.1 and 3.2. these assignments determine an $\operatorname{Aut}(Q)$-orbit in $\operatorname{End}_{\mathcal{Y}}(Q)$, and all orbits occur in this way. However, a permutation of the indices within a subset $A \in \mathcal{A}$ yields the same orbit. Thus, each orbit giving rise to this particular $\mathcal{A}$ is obtained by $\prod_{A \in \mathcal{A}}(|A| !)$ such assignments. Hence, we must divide by this number to obtain the number of orbits.

Now let $\mathbf{l}=\left(l_{1}, \ldots, l_{s}\right)$ be a tuple of nonnegative integers, and consider the quiver $R_{\mathbf{l}}(q)$. If $\mathbf{l}^{\prime}=\left(l_{1}^{\prime}, \ldots, l_{s}^{\prime}\right)$ is another such $s$-tuple, let $\mathbf{l}+\mathbf{l}^{\prime}=\left(l_{1}+l_{1}^{\prime}, \ldots, l_{s}+\right.$ $\left.l_{s}^{\prime}\right)$; write $\mathbf{l} \leq \mathbf{l}^{\prime}$ if $l_{i} \leq l_{i}^{\prime}$ for all $i$. Also, if $b \in \mathbb{Q}$, let $b \mathbf{l}=\left(b l_{1}, \ldots, b l_{s}\right)$. It is easy to see that all decompositions of $R_{1}$ into direct sums of subrepresentations are of the form

$$
R_{\mathbf{l}}=\bigoplus_{i=1}^{n} R_{\mathbf{l}^{i}}
$$

where $\mathbf{l}=\mathbf{l}^{1}+\cdots+\mathbf{l}^{n}$. Let $d \in \mathbb{N}$. If not all $l_{i}$ are divisible by $d$, then there is no $\mathbb{F}_{q^{d}}$-expansion of $R_{\mathbf{l}}(q)$. If all $l_{i}$ are divisible by $d$, then $R_{\mathbf{l}}(q)$ has a unique (up to the action of $\left.\operatorname{Aut}\left(R_{\mathrm{l}}(q)\right)\right) \mathbb{F}_{q^{d}}$-expansion, namely $R_{\mathbf{l} / d}\left(q^{d}\right)$.

Consider the set of tuples $J=\left(\mathbf{l}^{1}, \ldots, \mathbf{l}^{n}, d_{1}, \ldots, d_{n}\right)$ such that each $\mathbf{l}^{i}$ is an $s$-tuple of nonnegative integers, not all equal to zero, $d_{i} \in \mathbb{N}$ for each $i$ and

$$
\sum_{i=0}^{n} d_{i} \mathbf{l}^{i}=\mathbf{l}
$$

Let $\mathscr{D}(\mathbf{l})$ be a complete set of representatives of the action of the symmetric group $S_{n}$ on this set, where the action is by permuting the indices $1, \ldots, n$. Less formally, $\mathscr{D}(\mathbf{l})$ is in a one-to-one correspondence with ways to decompose $R_{\mathbf{l}}(q)$ as a direct sum of quivers $R_{\mathbf{1}^{\prime}}(q)$ and to expand each $R_{\mathbf{1}^{\prime}}(q)$ to $R_{\mathbf{1}^{\prime} / d}\left(q^{d}\right)$ for some $d$.

By Lemma 2.1, the numbers $\gamma\left(P^{\mathbf{l}}(q), P^{\mathbf{l}}(q)\right)$ and $\gamma\left(P^{\mathbf{l}}(q), M^{\mathbf{l}}(q)\right)$ may both be expressed as $\gamma\left(\operatorname{Aut}\left(R_{\mathbf{l}}(q)\right)\right.$, End $\left.\mathcal{Y}\left(R_{\mathbf{l}}(q)\right)\right)$ where $\mathcal{Y}$ is the class of all invertible endomorphisms or the class of all endomorphisms, respectively. If $\mathcal{Y}$ is one of those classes, then $k \mathcal{Y}, d(q)$ is polynomial in $q$. We deduce the following from Proposition 3.3 
Corollary 3.4. Let $\mathbf{l}=\left(l_{1}, \ldots, l_{s}\right)$ be a tuple of nonnegative integers. Then there exist tuples $\left(a_{J}\right)_{J \in \mathscr{D}(\mathbf{1})}$ and $\left(b_{J}\right)_{J \in \mathscr{D}(\mathbf{1})}$ where $a_{J}(T)$ and $b_{J}(T)$ are polynomials with rational coefficients such that, for all prime powers $q$,

$$
\begin{aligned}
\gamma\left(P^{\mathbf{l}}(q), P^{\mathbf{l}}(q)\right) & =\sum_{J \in \mathscr{D}(\mathbf{l})} a_{J}(q) \prod_{i=1}^{n} \rho_{\mathbf{l}^{i}}\left(q^{d_{i}}\right) \quad \text { and } \\
\gamma\left(P^{\mathbf{l}}(q), M^{\mathbf{l}}(q)\right) & =\sum_{J \in \mathscr{D}(\mathbf{l})} b_{J}(q) \prod_{i=1}^{n} \rho_{\mathbf{l}^{i}}\left(q^{d_{i}}\right)
\end{aligned}
$$

In each case, the first sum is over all elements $J=\left(\mathbf{l}^{1}, \ldots, \mathbf{l}^{n}, d_{1}, \ldots, d_{n}\right)$ of $\mathscr{D}(\mathbf{l})$.

\section{Preliminary results}

In this section we state several standard and straightforward results. We prove the last of these results; the first two are easy exercises.

Lemma 4.1. Let $G$ be a group acting on a set $Y$. Let $N$ be a normal subgroup of $G$, and let $S$ be the set of $N$-orbits on $Y$. The action of $G$ on $Y$ induces an action of $G / N$ on $S$ via $g N \circ N y=N g y$ (for all $g \in G, y \in Y$ ). Moreover, the $G$-orbits on $Y$ are in a one-to-one correspondence with the $G / N$-orbits on $S$.

Lemma 4.2. Let $G$ be a group acting on finite sets $X$ and $Y$. Suppose $S$ is a subset of $X \times Y$ preserved by $G$. Let $R$ be a complete set of representatives of $G$-orbits on $X$ (so each $G$-orbit on $X$ contains exactly one element of $R$ ). Then

$$
\gamma(G, S)=\sum_{x \in R} \gamma\left(\operatorname{Stab}_{G}(x),\{y \in Y:(x, y) \in S\}\right) .
$$

Recall that if $V$ and $W$ are vector spaces and $X \in \operatorname{End}(V), Y \in \operatorname{End}(W)$, then

$$
\mathcal{I}(X, Y)=\{T \in \operatorname{Hom}(V, W): T X=Y T\} .
$$

Lemma 4.3. Let $V$ and $W$ be finite dimensional vector spaces over a field $K$. Suppose $X \in \operatorname{End}(V)$ and $Y \in \operatorname{End}(W)$. Then

$$
\operatorname{dim} \mathcal{I}(X, Y)=\operatorname{dim} \mathcal{I}(Y, X) .
$$

Proof. Let $V^{*}$ and $W^{*}$ be the dual spaces to $V$ and $W$. Let $X^{*} \in \operatorname{End}\left(V^{*}\right)$ and $Y^{*} \in \operatorname{End}\left(W^{*}\right)$ be the maps dual to $X$ and $Y:\left(X^{*} f\right)(v)=f(X(v))$ for all $f \in V^{*}, v \in V$. Then $\operatorname{dim} \mathcal{I}(Y, X)=\operatorname{dim} \mathcal{I}\left(X^{*}, Y^{*}\right)$ : a linear bijection between $\mathcal{I}(Y, X)$ and $\mathcal{I}\left(X^{*}, Y^{*}\right)$ is given by assigning to each map its dual. Also, $X^{*}$ and $X$ have the same rational canonical form, as do $Y$ and $Y^{*}$. Thus,

$$
\operatorname{dim} \mathcal{I}(X, Y)=\operatorname{dim} \mathcal{I}\left(X^{*}, Y^{*}\right)=\operatorname{dim} \mathcal{I}(Y, X) .
$$

\section{Action on the dual space}

Let $V$ be a finite dimensional vector space over a finite field $\mathbb{F}_{q}$. If $w \in V^{*}$ and $v \in V$, we shall write $(w, v)$ for $w(v)$. Suppose a group $G$ acts on $V$ by linear maps, so a representation of $G$ on $V$ is given:

$$
(g, v) \mapsto g v .
$$


This action gives rise to a representation of $G$ on $V^{*}$ in the usual way:

$$
\left((g w, v)=\left(w, g^{-1} v\right),\right.
$$

where $g \in G, w \in V^{*}, v \in V$. We shall rely on the following simple result, which is a weak version of Brauer's Theorem ([3, 11.9]).

Lemma 5.1. The number of $G$-orbits on $V$ is equal to the number of $G$-orbits on $V^{*}$.

Proof. By a well-known orbit-counting formula,

$$
\gamma(G, V)=\frac{1}{|G|} \sum_{g \in G} \operatorname{Fix}_{V}(g),
$$

where $\operatorname{Fix}_{V}(g)$ is the number of elements of $V$ fixed by $g$. The same holds for $V^{*}$. Therefore, it suffices to show that $\operatorname{Fix}_{V}(g)=\operatorname{Fix}_{V^{*}}(g)$ for all $g \in G$.

Let $g \in G$. Fix a basis of $V$, and let $A$ be the matrix of the action of $g$ with respect to this basis. Then $\left(A^{t}\right)^{-1}$ is the matrix of the action of $g$ on $V^{*}$ with respect to the dual basis. Since $\operatorname{rank}(A-I)=\operatorname{rank}\left(A^{t}-I\right)=\operatorname{rank}\left(\left(A^{t}\right)^{-1}-I\right)$,

$$
\operatorname{Fix}_{V}(g)=q^{n-\operatorname{rank}(A-I)}=q^{n-\operatorname{rank}\left(\left(A^{t}\right)^{-1}-I\right)}=\operatorname{Fix}_{V^{*}}(g) .
$$

Let $Q=\left(E_{0}, E_{1}, \mathbf{U}, \boldsymbol{\alpha}\right)$ be a quiver representation. Let $a, b \in E_{0}$. Define an extension $\Omega(Q, a, b)=Q^{\prime}=\left(E_{0}^{\prime}, E_{1}^{\prime}, \mathbf{U}^{\prime}, \boldsymbol{\alpha}^{\prime}\right)$ of $Q$ as follows:

(i) $E_{0}^{\prime}=E_{0} \sqcup\{c\}$;

(ii) $\operatorname{dim} U_{c}^{\prime}=\operatorname{dim} U_{a}+\operatorname{dim} U_{b}$;

(iii) $E_{1}^{\prime}=E_{1} \sqcup\left\{e_{1}, e_{2}\right\}$ where $\sigma\left(e_{1}\right)=a, \tau\left(e_{1}\right)=c=\sigma\left(e_{2}\right), \tau\left(e_{2}\right)=b$;

(iv) $\alpha_{e}^{\prime}=\alpha_{e}$ for all $e \in E_{1}$;

(v) $\alpha_{e_{1}}$ is injective, $\alpha_{e_{2}}$ is surjective, and $\operatorname{im}\left(\alpha_{e_{1}}\right)=\operatorname{ker}\left(\alpha_{e_{2}}\right)$, so the sequence

$$
0 \longrightarrow U_{a} \stackrel{\alpha_{e_{1}}}{\longrightarrow} U_{c}^{\prime} \stackrel{\alpha_{e_{2}}}{\longrightarrow} U_{b} \longrightarrow 0
$$

is exact.

Write $\pi$ for $\pi_{Q}^{Q^{\prime}}: \operatorname{End}\left(Q^{\prime}\right) \rightarrow \operatorname{End}(Q)$. Note that $\operatorname{Aut}(Q)$ acts on $\operatorname{Hom}\left(X_{a}, X_{b}\right)$ in a natural way: $\mathbf{g} \circ R=g_{b} R g_{a}^{-1}\left(\mathrm{~g} \in \operatorname{Aut}(Q), R \in \operatorname{Hom}\left(X_{a}, X_{b}\right)\right)$.

Proposition 5.2. If $\mathbf{X}$ is an endomorphism of $Q$ and $H$ is a subgroup of $\operatorname{Aut}(Q)$ that fixes $\mathbf{X}$, then

$$
\gamma\left(H^{Q^{\prime}}, \pi^{-1}(\mathbf{X})\right)=\gamma\left(H, \mathcal{I}\left(X_{a}, X_{b}\right)\right) .
$$

Proof. Let $k=\operatorname{dim} U_{a}, m=\operatorname{dim} U_{b}$. Let $\beta=\alpha_{e_{1}}$. Let $V=\beta\left(U_{a}\right) \leq U_{c}^{\prime}$. Choose a complement $W$ of $V$ in $U_{c}^{\prime}$, so that $U_{c}^{\prime}=V \oplus W$. Then $\epsilon:=\left.\alpha_{e_{2}}\right|_{W}$ is a bijective linear map from $W$ onto $U_{b}$.

For any $R \in \operatorname{Hom}\left(U_{b}, U_{a}\right)$, let $\phi(R)$ be the unique element of $\pi^{-1}(\mathbf{X})$ such that, for $v \in V, w \in W$,

$$
\phi(R)_{c}(v+w)=\beta X_{a} \beta^{-1} \cdot v+\epsilon^{-1} X_{b} \epsilon \cdot w+\beta R \epsilon \cdot w .
$$

Less formally, if one chooses bases for $U_{a}$ and $U_{b}$, uses $\beta$ and $\epsilon^{-1}$ to get bases of $V$ and $W$, combines these to get a basis of $U_{c}^{\prime}$ and represents linear operators as matrices using these bases, then

$$
\phi(R)_{c}=\left(\begin{array}{cc}
X_{a} & R \\
0 & X_{b}
\end{array}\right)
$$


Clearly, $\phi$ is a bijection from $\operatorname{Hom}\left(U_{b}, U_{a}\right)$ onto $\pi^{-1}(\mathbf{X})$. For $T \in \operatorname{Hom}\left(U_{b}, U_{a}\right)$, define $\psi(T) \in H^{Q^{\prime}}$ as follows: $\psi(T)_{d}=I_{U_{d}}$ for $d \neq c$, and

$$
\psi(T)_{c}(v+w)=v+w+\beta T \epsilon \cdot w \quad \forall v \in V, w \in W .
$$

As a matrix,

$$
\psi(T)_{c}=\left(\begin{array}{cc}
I_{k} & T \\
0 & I_{m}
\end{array}\right) .
$$

Consider the surjective group homomorphism $\pi^{\prime}:=\left.\pi\right|_{H^{Q^{\prime}}}: H^{Q^{\prime}} \rightarrow H$. Let $A=\operatorname{ker} \pi^{\prime}$. Then $\psi: \operatorname{Hom}\left(U_{b}, U_{a}\right) \rightarrow A$ is an isomorphism of abelian groups. A direct calculation (e.g. using matrices) produces a formula for the action of $A$ on $\pi^{-1}(\mathbf{X})$ :

$$
\psi(T) \phi(R) \psi(T)^{-1}=\phi\left(R+T X_{b}-X_{a} T\right) .
$$

Let

$$
D=\left\{T X_{b}-X_{a} T: T \in \operatorname{Hom}\left(U_{b}, U_{a}\right)\right\} .
$$

Then the map $\phi^{-1}: \pi^{-1}(\mathbf{X}) \rightarrow \operatorname{Hom}\left(U_{b}, U_{a}\right)$ establishes a one-to-one correspondence between $A$-orbits on $\pi^{-1}(\mathbf{X})$ and cosets of $D$ in $\operatorname{Hom}\left(U_{b}, U_{a}\right)$. Hence, the maps $\pi^{\prime}$ and $\phi$ establish an isomorphism between the action of $H^{Q^{\prime}} / A$ on the set of $A$-orbits in $\pi^{-1}(\mathbf{X})$ and the natural action of $H$ on $\operatorname{Hom}\left(U_{b}, U_{a}\right) / D$. It follows, by Lemma 4.1 (applied to the normal subgroup $A$ of $H^{Q^{\prime}}$ ), that

$$
\gamma\left(H^{Q^{\prime}}, \pi^{-1}(\mathbf{X})\right)=\gamma\left(H, \operatorname{Hom}\left(U_{b}, U_{a}\right) / D\right) .
$$

The dual space to $\operatorname{Hom}\left(U_{b}, U_{a}\right)$ may be identified with $\operatorname{Hom}\left(U_{a}, U_{b}\right)$, where

$$
(S, R)=\operatorname{tr}(S R) \quad \forall R \in \operatorname{Hom}\left(U_{b}, U_{a}\right), S \in \operatorname{Hom}\left(U_{a}, U_{b}\right),
$$

and the dual action of $H$ on the space $\operatorname{Hom}\left(U_{a}, U_{b}\right)$ is induced by the natural action of $\operatorname{GL}\left(U_{a}\right) \times \mathrm{GL}\left(U_{b}\right)$ on this space. Hence the dual space of $\operatorname{Hom}\left(U_{b}, U_{a}\right) / D$ is

$$
D^{\perp}=\left\{S \in \operatorname{Hom}\left(U_{a}, U_{b}\right): \operatorname{tr}(R S)=0 \text { for all } R \in D\right\} .
$$

Any $S \in \mathcal{I}\left(X_{a}, X_{b}\right)$ belongs to $D^{\perp}$. Indeed, for all $T \in \operatorname{Hom}\left(U_{b}, U_{a}\right)$,

$$
\begin{aligned}
\operatorname{tr}\left(S\left(T X_{b}-X_{a} T\right)\right) & =\operatorname{tr}\left(S T X_{b}\right)-\operatorname{tr}\left(S X_{a} T\right)=\operatorname{tr}\left(X_{b} S T\right)-\operatorname{tr}\left(S X_{a} T\right) \\
& =\operatorname{tr}\left(\left(X_{b} S-S X_{a}\right) T\right)=0 .
\end{aligned}
$$

Moreover,

$$
\operatorname{dim} D^{\perp}=k m-\operatorname{dim} D=\operatorname{dim} \mathcal{I}\left(X_{b}, X_{a}\right)=\operatorname{dim} \mathcal{I}\left(X_{a}, X_{b}\right) .
$$

(The last equality holds by Lemma 4.3.) It follows that $D^{\perp}=\mathcal{I}\left(X_{a}, X_{b}\right)$. By Lemma 5.1

$$
\gamma\left(H, \operatorname{Hom}\left(U_{b}, U_{a}\right) / D\right)=\gamma\left(H, D^{\perp}\right)=\gamma\left(H, \mathcal{I}\left(X_{a}, X_{b}\right)\right),
$$

and the result follows.

Proposition 5.2 will allow us to replace the quiver $Q^{\prime}=\Omega(Q, a, b)$ with quivers obtained from $Q$ by adding an arrow from $U_{a}$ to $U_{b}$ when counting Aut $\left(Q^{\prime}\right)$-orbits. A more usable form of this proposition is given by the corollary below.

Let $Q=\left(E_{0}, E_{1}, \mathbf{U}, \boldsymbol{\alpha}\right), a, b \in E_{0}, Q^{\prime}=\Omega(Q, a, b), \pi=\pi_{Q}^{Q^{\prime}}$ be as above. Let $G$ be a subgroup of $\operatorname{Aut}(Q)$. Let $\Xi=\Xi(Q, G, a, b)$ be a complete set of representatives of $G$-orbits on $\operatorname{Hom}\left(U_{a}, U_{b}\right)$ (the choice of $\Xi$ does not matter). For each $S \in \Xi$, let $Q^{S}$ be the quiver $\left(E_{0}, E_{1} \sqcup\{f\}, \mathbf{U}, \alpha^{S}\right)$ where $\sigma(f)=a$, $\tau(f)=b, \alpha_{e}^{S}=\alpha_{e}$ for $e \in E_{1}$, and $\alpha_{f}^{S}=S$. That is, $Q^{S}$ is obtained from $Q$ by adding the linear map $S: U_{a} \rightarrow U_{b}$. 
Corollary 5.3. Let $B$ be a subset of $\operatorname{End}(Q)$ preserved by a subgroup $G$ of $\operatorname{Aut}(Q)$. Then

$$
\gamma\left(G^{Q^{\prime}}, \pi^{-1}(B)\right)=\sum_{S \in \Xi} \gamma\left(G \cap \operatorname{Aut}\left(Q^{S}\right), B \cap \operatorname{End}\left(Q^{S}\right)\right) .
$$

Proof. Let $Y$ be a complete set of representatives of $G$-orbits on $B$. Let

$$
Z=\left\{(S, \mathbf{X}) \in \operatorname{Hom}\left(U_{a}, U_{b}\right) \times B: S X_{a}=X_{b} S\right\} .
$$

Then

$$
\begin{aligned}
\gamma\left(G^{Q^{\prime}}, \pi^{-1}(B)\right) & =\sum_{\mathbf{X} \in Y} \gamma\left(\operatorname{Stab}_{G}(\mathbf{X})^{Q^{\prime}}, \pi^{-1}(\mathbf{X})\right) \\
& =\sum_{\mathbf{X} \in Y} \gamma\left(\operatorname{Stab}_{G}(\mathbf{X}), \mathcal{I}\left(X_{a}, X_{b}\right)\right) \\
& =\gamma(G, Z)=\sum_{S \in \Xi} \gamma\left(G \cap \operatorname{Aut}\left(Q^{S}\right), B \cap \operatorname{End}\left(Q^{S}\right)\right) .
\end{aligned}
$$

The first equality holds by Lemma 4.2, applied to the set

$$
\left\{\left(\mathbf{X}, \mathbf{X}^{\prime}\right) \in B \times \operatorname{End}\left(Q^{\prime}\right): \pi\left(\mathbf{X}^{\prime}\right)=\mathbf{X}\right\} .
$$

The second one follows from Proposition 5.2. The third and fourth equalities follow from Lemma 4.2, applied to the set $Z$ in two different ways.

The rest of this section is devoted to an informal sketch of a proof of Theorem 1.1 in the special case when $\mathcal{Y}$ is the class of nilpotent matrices. A formal proof of a more general statement is in the next section. Let $Q$ be the quiver representation consisting of vector spaces $U_{a}=\mathbb{F}_{q}^{k}$ and $U_{b}=\mathbb{F}_{q}^{m}$ without any arrows. Let $Q^{\prime}=\Omega(Q, a, b)$, so $Q^{\prime}$ is the quiver representation

$$
\mathbb{F}_{q}^{k C} \longrightarrow \mathbb{F}_{q}^{k+m} \longrightarrow \mathbb{F}_{q}^{m}
$$

where the first map is injective, the second one is surjective, and the sequence is exact at $\mathbb{F}_{q}^{k+m}$. It is not difficult to see that $\rho_{(k, m)}(q)=\theta\left(Q^{\prime}\right)$. The orbit of an element $S_{1} \in \operatorname{Hom}\left(\mathbb{F}_{q}^{k}, \mathbb{F}_{q}^{m}\right)$ with respect to the action of $\operatorname{Aut}(Q)=\mathrm{GL}_{k}(q) \times$ $\mathrm{GL}_{m}(q)$ is determined by $\operatorname{rank}\left(S_{1}\right)$. Thus, by Corollary 5.3 (applied to $G=$ Aut $(Q)$ and $B=N(Q)), \rho_{(k, m)}(q)=\theta\left(Q^{\prime}\right)$ may be expressed as a sum, with one summand for each possible value of $\operatorname{rank}\left(S_{1}\right)$. If $S_{1}=0$, we get the summand $\theta(Q)=p(k) p(m)$. Otherwise, the summand corresponding to $S_{1}$ is $\theta\left(Q_{1}\right)$ where $Q_{1}$ is the quiver representation

$$
\mathbb{F}_{q}^{k} \stackrel{S_{1}}{\longrightarrow} \mathbb{F}_{q}^{m}
$$

Let $\lambda_{1}=\operatorname{rank} S_{1}$. Then $\operatorname{ker} S_{1}$ and $\operatorname{im} S_{1}$ may be identified with $\mathbb{F}_{q}^{k-\lambda_{1}}$ and $\mathbb{F}_{q}^{\lambda_{1}}$ respectively. It follows from Lemma 2.2 that $\theta\left(Q_{1}\right)=\theta\left(\bar{Q}_{1}\right)$ where $\bar{Q}_{1}$ is the quiver representation

$$
\mathbb{F}_{q}^{k-\lambda_{1}} \longrightarrow \mathbb{F}_{q}^{k} \stackrel{S_{1}}{\longrightarrow} \mathbb{F}_{q}^{\lambda_{1}} \longrightarrow \mathbb{F}_{q}^{m}
$$

with the sequence exact at $\mathbb{F}_{q}^{k}$. We apply Corollary 5.3 again: $\theta\left(\bar{Q}_{1}\right)$ may be expressed as a sum of terms corresponding to maps $S_{2} \in \operatorname{Hom}\left(\mathbb{F}_{q}^{k-\lambda_{1}}, \mathbb{F}_{q}^{\lambda_{1}}\right)$ of 
varying ranks. (Again, we have one map of each possible rank.) Thus, we branch into cases corresponding to the possible values of $\lambda_{2}:=\operatorname{rank} S_{2}$.

We continue this argument inductively, considering maps $S_{3}, S_{4}, \ldots$ of ranks $\lambda_{i}:=\operatorname{rank} S_{i}$ until $S_{s+1}=0$ for some $s$. (Here, $S_{i+1}: \mathbb{F}_{q}^{k-\lambda_{1}-\cdots-\lambda_{i}} \rightarrow \mathbb{F}_{q}^{\lambda_{i}}$.) Ultimately, we express $\theta\left(Q^{\prime}\right)=\rho_{(k, m)}(q)$ as a sum of terms indexed by partitions $\lambda=\left(\lambda_{1}, \ldots, \lambda_{s}\right)$. The term corresponding to $\lambda$ is equal to $\theta\left(Q_{s}\right)$ where $Q_{s}$ is the quiver representation

$$
\mathbb{F}_{q}^{k-|\lambda|} \quad \mathbb{F}_{q}^{\lambda_{s}} \longrightarrow \mathbb{F}_{q}^{\lambda_{s-1}} \longleftrightarrow \cdots \hookrightarrow \mathbb{F}_{q}^{\lambda_{1}} \longrightarrow \mathbb{F}_{q}^{m} .
$$

It follows from Lemma 2.1 that $\theta\left(Q_{s}\right)=p(k-|\lambda|) \nu_{\lambda}^{m}(q)$. Putting $j=|\lambda|$, we obtain

$$
\rho_{(k, m)}(q)=\sum_{j=0}^{k} p(k-j) \sum_{\lambda \in \mathcal{P}_{j}^{m}} \nu_{\lambda}^{m}(q),
$$

as stated in Theorem 1.1 .

\section{An inductive argument}

Fix a positive integer $m$. Let $W$ be an $m$-dimensional vector space over $\mathbb{F}_{q}$. Let $G$ be a subgroup of $\mathrm{GL}(W)$, and let $A \subseteq \operatorname{End}(W)$ be a set preserved by $G$.

If $\lambda=\left(\lambda_{1}, \ldots, \lambda_{s}\right) \in \mathcal{P}^{m}$, let $\mathcal{F}_{\lambda}$ be the set of flags $\mathbf{W}=\left(W_{1}, W_{2}, \ldots, W_{s}\right)$ such that

$$
W \geq W_{1} \geq W_{2} \geq \cdots \geq W_{s}>0
$$

and $\operatorname{dim} W_{i}=\lambda_{i}$. If $\mathbf{W}=\left(W_{1}, \ldots, W_{s}\right) \in \mathcal{F}_{\lambda}$ and $\mathbf{W}^{\prime}=\left(W_{1}, \ldots, W_{s+1}\right) \in \mathcal{F}_{\lambda^{\prime}}$ for an appropriate $\lambda^{\prime}=\left(\lambda_{1}, \ldots, \lambda_{s+1}\right)$ (that is, the first $s$ subspaces are the same for $\mathbf{W}$ and $\mathbf{W}^{\prime}$ ), we shall write $\mathbf{W}^{\prime} \succ \mathbf{W}$. For each $\lambda \in \mathcal{P}^{m}$, choose a complete set $F_{\lambda}$ of representatives of $G$-orbits on $\mathcal{F}_{\lambda}$. We may assume that these choices satisfy the following property: if $\mathbf{W} \in \mathcal{F}_{\lambda}, \mathbf{W}^{\prime} \in F_{\lambda^{\prime}}$ and $\mathbf{W}^{\prime} \succ \mathbf{W}$, then $\mathbf{W} \in F_{\lambda^{\prime}}$. Let $\lambda \in \mathcal{P}^{m}$. Let

$$
\begin{gathered}
T_{\lambda}(A)=\left\{(\mathbf{W}, R) \in \mathcal{F}_{\lambda} \times A: R\left(W_{i}\right) \subseteq W_{i} \text { for all } i\right\}, \text { and let } \\
\xi_{\lambda}(G, A)=\gamma\left(G, T_{\lambda}(A)\right) .
\end{gathered}
$$

Let $\lambda=\left(\lambda_{1}, \ldots, \lambda_{s}\right) \in \mathcal{P}^{m}$, and let $\mathbf{W}=\left(W_{1}, \ldots, W_{s}\right) \in \mathcal{F}_{\lambda}$. Let $Q_{\lambda}(\mathbf{W})$ be the quiver representation

$$
W_{s} \longrightarrow W_{s-1} \longrightarrow \cdots \hookrightarrow W_{1} \longrightarrow W
$$

where the arrows are the inclusion maps (obtained by restricting $I_{W}$ ). If $s=0$ (so $\lambda=()$ ), we get the quiver representation $Q_{()}$consisting just of the space $W$ without any arrows.

Let $\mathcal{Y}$ be a class of linear operators over $\mathbb{F}_{q}$. We shall assume that $A \subseteq \mathcal{Y}$. Let $Q$ be an extension of $Q_{()}$. Let $\pi=\pi_{Q_{(}}^{Q}: \operatorname{End}(Q) \rightarrow \operatorname{End}\left(Q_{()}\right)$be the natural projection map. Recall that

$$
\begin{aligned}
G^{Q} & =\pi^{-1}(G) \cap \operatorname{Aut}(Q), \quad \text { and let } \\
A_{\mathcal{Y}}^{Q} & =\pi^{-1}(A) \cap \operatorname{End}_{\mathcal{Y}}(Q) .
\end{aligned}
$$

By Lemma 2.1, for any $\mathbf{W} \in \mathcal{F}_{\lambda}, \operatorname{Stab}_{G}(\mathbf{W})$-orbits on $\left\{R \in A: R\left(W_{i}\right) \subseteq\right.$ $\left.W_{i} \forall i\right\}$ are in a one-to-one correspondence with $G^{Q_{\lambda}(\mathbf{W})}$-orbits on $A^{Q_{\lambda}(\mathbf{W})}$. It follows, by Lemma 4.2, that

$$
\xi_{\lambda}(G, A)=\sum_{\mathbf{W} \in F_{\lambda}} \gamma\left(G^{Q_{\lambda}(\mathbf{W})}, A^{Q_{\lambda}(\mathbf{W})}\right) .
$$


Let $\mathbf{W} \in \mathcal{F}_{\lambda}$ and $k \in \mathbb{Z}_{\geq 0}$. Let $D_{\lambda}^{k}(\mathbf{W})$ be the quiver representation

$$
V \quad W_{s} \longrightarrow W_{s-1} \longleftrightarrow \cdots \hookrightarrow W_{1} \longrightarrow W
$$

where $\operatorname{dim} V=k$ and the maps are as in $Q_{\lambda}(\mathbf{W})$ (i.e. injective). Let $Q_{\lambda}^{k}(\mathbf{W})$ be the quiver representation $\Omega\left(D_{\lambda}^{k}(\mathbf{W}), a, b\right)$ (as defined in the previous section) where $a$ and $b$ correspond to $V$ and $W_{s}$ respectively. Then $Q_{\lambda}^{k}(\mathbf{W})$ may be depicted as

$$
V^{\longrightarrow} Z \longrightarrow W_{s} \longrightarrow W_{s-1} \longleftrightarrow \cdots \hookrightarrow W_{1} \longrightarrow W
$$

(Note that $\operatorname{dim} Z=k+\lambda_{s}$ and the sequence is exact at $Z$.) Consider again the case $\lambda=($ ). (Then $\mathbf{W}$ is the empty sequence and is omitted from the notation.) The corresponding quiver representation $D_{()}^{k}$ does not have any arrows and consists just of the vector spaces $V(\operatorname{dim} V=k)$ and $W$. The quiver representation $Q_{()}^{k}$ is

$$
V \hookrightarrow Z \longrightarrow W
$$

where the sequence is exact at $Z$. Write $D^{k}$ for $D_{()}^{k}$ and $Q^{k}$ for $Q_{()}^{k}$. Let $G^{(k)}=G^{Q^{k}}$ and $A_{\mathcal{Y}}^{(k)}=A_{\mathcal{Y}}^{Q^{k}}$. Recall that $c(k, \mathcal{Y})$ is the number of $\operatorname{GL}(V)$ orbits on $\operatorname{End}_{\mathcal{Y}}(V)$. We shall prove the following result using Corollary 5.3 ,

Lemma 6.1. Let $\lambda=\left(\lambda_{1}, \ldots, \lambda_{s}\right) \in \mathcal{P}^{m}$ and $k \in \mathbb{Z}_{\geq 0}$. Let $\mathbf{W} \in F_{\lambda}$. Then

$$
\begin{aligned}
\gamma\left(G^{Q_{\lambda}^{k}(\mathbf{W})}, A_{\mathcal{Y}}^{Q_{\lambda}^{k}(\mathbf{W})}\right) & =c(k, \mathcal{Y}) \gamma\left(G^{Q_{\lambda}(\mathbf{W})}, A_{\mathcal{Y}}^{Q_{\lambda}(\mathbf{W})}\right) \\
& +\sum_{\lambda_{s+1}=1}^{\min \left(\lambda_{s}, k\right)} \sum_{\substack{\mathbf{W}^{\prime} \in F_{\lambda^{\prime}} \\
\mathbf{W}^{\prime} \succ \mathbf{W}}} \gamma\left(G^{Q_{\lambda}^{k-\lambda_{s+1}}\left(\mathbf{W}^{\prime}\right)}, A_{\mathcal{Y}}^{Q_{\lambda}^{k-\lambda_{s+1}}\left(\mathbf{W}^{\prime}\right)}\right)
\end{aligned}
$$

where $\lambda^{\prime}=\left(\lambda_{1}, \ldots, \lambda_{s}, \lambda_{s+1}\right)$.

Proof. Let $\Phi$ be the set of subspaces $W_{s+1}$ of $W_{s}$ such that

$$
\mathbf{W}^{\prime}:=\left(W_{1}, \ldots, W_{s}, W_{s+1}\right) \in F_{\left(\lambda_{1}, \ldots, \lambda_{s}, \lambda_{s+1}\right)}
$$

for some $\lambda_{s+1} \in[1, k]$ (of course, then $\operatorname{dim} W_{s+1}=\lambda_{s+1}$ ). Then $\Phi$ is a complete set of representatives of $\operatorname{Stab}_{G}(\mathbf{W})$ on non-zero subspaces of $W_{s}$ of dimension at most $k$. For each $W_{s+1} \in \Phi$, choose a linear map $\alpha_{W_{s+1}}: V \rightarrow W_{s}$ with image $W_{s+1}$.

Let $H=G^{D_{\lambda}^{k}(\mathbf{W})}$. Then $H=\mathrm{GL}(V) \times G^{Q_{\lambda}(\mathbf{W})}$. Thus, any two elements of $\operatorname{Hom}\left(V, W_{s}\right)$ are $H$-conjugate if and only if their images in $W_{s}$ are $\operatorname{Stab}_{G}(\mathbf{W})$ conjugate. Let $\Xi$ be the set consisting of the zero map $V \rightarrow W_{s}$ and all the maps $\alpha_{W_{s+1}}$ where $W_{s+1}$ runs through $\Phi$. Then $\Xi$ is a complete set of representatives of $H$-orbits on $\operatorname{Hom}\left(V, W_{s}\right)$. For each $\alpha \in \Xi$, let $L^{\alpha}$ be the quiver obtained from $D_{\lambda}^{k}(\mathbf{W})$ by adding the map $\alpha: V \rightarrow W_{s}$, that is,

$$
V \stackrel{\alpha}{\longrightarrow} W_{s} \longrightarrow W_{s-1} \longrightarrow \cdots \hookrightarrow W_{1} \longrightarrow W .
$$

By Corollary 5.3, applied to the group $G^{D_{\lambda}^{k}(\mathbf{W})} \leq \operatorname{Aut}\left(D_{\lambda}^{k}(\mathbf{W})\right)$ and to the set $A_{\mathcal{Y}}^{D_{\lambda}^{k}(\mathbf{W})} \subseteq \operatorname{End}\left(D_{\lambda}^{k}(\mathbf{W})\right)$,

$$
\gamma\left(G^{Q_{\lambda}^{k}(\mathbf{W})}, A_{\mathcal{Y}}^{Q_{\lambda}^{k}(\mathbf{W})}\right)=\sum_{\alpha \in \Xi} \gamma\left(G^{L^{\alpha}}, A_{\mathcal{Y}}^{L^{\alpha}}\right)
$$


(Here we use the fact that, if $\mathbf{X} \in \operatorname{End}\left(Q_{\lambda}^{k}(\mathbf{W})\right)$ and the actions of $\mathbf{X}$ on $V$ and $W_{s}$ are $\mathcal{Y}$-endomorphisms, then so is the action of $\mathbf{X}$ on $Z$.)

If $\alpha=0$, then, obviously,

$$
\gamma\left(G^{L^{\alpha}}, A_{\mathcal{Y}}^{L^{\alpha}}\right)=\gamma\left(G^{D_{\lambda}^{k}(\mathbf{W})}, A_{\mathcal{Y}}^{D_{\lambda}^{k}(\mathbf{W})}\right) .
$$

Since the quiver representation $D_{\lambda}^{k}(\mathbf{W})$ is the disconnected union of $Q_{\lambda}(\mathbf{W})$ and the quiver representation that consists of the space $V$, we have

$$
\gamma\left(G^{L^{0}}, A_{\mathcal{Y}}^{L^{0}}\right)=c(k, \mathcal{Y}) \gamma\left(G^{Q_{\lambda}(\mathbf{W})}, A_{\mathcal{Y}}^{Q_{\lambda}(\mathbf{W})}\right) .
$$

Now consider the case $\alpha \neq 0$. Then $\alpha=\alpha_{W_{s+1}}$ for some $W_{s+1} \in F_{\lambda^{\prime}}$. Let $O$ be the quiver representation $I\left(K\left(L^{\alpha}, e\right), e\right)$ where $e$ is the arrow from $V$ to $W_{s}$ in $L^{\alpha}$. Then $O$ may be depicted as

$$
V^{\prime} \longrightarrow V \longrightarrow W_{s+1} \longrightarrow W_{s} \longrightarrow \cdots \hookrightarrow W_{1} \longrightarrow W
$$

where $V^{\prime}=\operatorname{ker} \alpha$ and the map $V \rightarrow W_{s+1}$ is induced by $\alpha$. By Lemma 2.2, the $G^{L^{\alpha}}$-orbits on $A_{\mathcal{Y}}^{L^{\alpha}}$ are in a one-to-one correspondence with the $G^{O}$-orbits on $A_{\mathcal{Y}}$. However, renaming $V$ as $Z$ and $V^{\prime}$ as $V$, we may identify $O$ with $Q_{\lambda^{\prime}}^{k-\lambda_{s+1}}(\mathbf{W})$ where $\lambda^{\prime}=\left(\lambda_{1}, \ldots, \lambda_{s}, \lambda_{s+1}\right)$. Thus,

$$
\gamma\left(G^{L^{\alpha}}, A_{\mathcal{Y}}^{L^{\alpha}}\right)=\gamma\left(G^{Q_{\lambda^{\prime}}^{k-\lambda_{s+1}}(\mathbf{W})}, A_{\mathcal{Y}^{\lambda^{\prime}}}^{Q^{k-\lambda_{s+1}}(\mathbf{W})}\right) .
$$

The result now follows from (3), (4) and (5).

The main result of this paper may be stated in a very general form as follows.

Theorem 6.2. Let $\mathcal{Y}$ be a class of linear operators over $\mathbb{F}_{q}$. Let $k \in \mathbb{N}$. Suppose that $G$ is a subgroup of $\mathrm{GL}(W)$ and that $A \subseteq \operatorname{End}(W) \cap \mathcal{Y}$ is preserved by $G$. Then

$$
\gamma\left(G^{(k)}, A_{\mathcal{Y}}^{(k)}\right)=\sum_{j=0}^{k} c(k-j, \mathcal{Y}) \sum_{\lambda \in \mathcal{P}_{j}^{m}} \xi_{\lambda}(G, A) .
$$

Proof. Recall that $l(\lambda)$ is the length (the number of parts) of a partition $\lambda$. If $s$ is a nonnegative integer, let

$$
\begin{aligned}
& a_{s}=\sum_{j=0}^{k} \sum_{\substack{\lambda \in \mathcal{P}_{j}^{m} \\
l(\lambda)=s}} c(k-j, \mathcal{Y}) \xi_{\lambda}(G, A), \\
& b_{s}=\sum_{j=0}^{k} \sum_{\substack{\lambda \in \mathcal{P}_{k-j}^{m} \\
l(\lambda)=s}} \sum_{\mathbf{W} \in F_{\lambda}} \gamma\left(G^{Q_{\lambda}^{j}(\mathbf{W})}, A_{\mathcal{Y}}^{Q_{\lambda}^{j}(\mathbf{W})}\right) .
\end{aligned}
$$

Since $A \subseteq \mathcal{Y}$, we have $A_{\mathcal{Y}}^{Q_{\lambda}(\mathbf{W})}=A^{Q_{\lambda}(\mathbf{W})}$. Thus, by (1),

$$
\xi_{\lambda}(G, A)=\sum_{\mathbf{W} \in F_{\lambda}} \gamma\left(G^{Q_{\lambda}(\mathbf{W})}, A_{\mathcal{Y}}^{Q_{\lambda}(\mathbf{W})}\right)
$$

Observe that

$$
b_{0}=\gamma\left(G^{(k)}, A_{\mathcal{Y}}^{(k)}\right)
$$


Indeed, the only non-zero summand of $b_{0}$ corresponds to the case $j=k, \lambda=()$. Applying Lemma 6.1 and rearranging the sums, we infer that, for any $s$,

$$
\begin{aligned}
b_{s} & =\sum_{j=0}^{k} \sum_{\substack{\lambda \in \mathcal{P}_{k-j}^{m} \\
l(\lambda)=s}} \sum_{\mathbf{W} \in F_{\lambda}} \gamma\left(G^{Q_{\lambda}^{j}(\mathbf{W})}, A_{\mathcal{Y}}^{Q_{\lambda}^{j}(\mathbf{W})}\right) \\
& =\sum_{j=0}^{k} \sum_{\substack{\lambda \in \mathcal{P}_{k-j}^{m} \\
l(\lambda)=s}} \sum_{\mathbf{W} \in F_{\lambda}} c(j, \mathcal{Y}) \gamma\left(G^{Q_{\lambda}(\mathbf{W})}, A_{\mathcal{Y}}^{Q_{\lambda}(\mathbf{W})}\right) \\
& +\sum_{i=0}^{k} \sum_{\substack{\lambda^{\prime} \in \mathcal{P}_{k-i}^{m} \\
l\left(\lambda^{\prime}\right)=s+1}} \sum_{\mathbf{W}^{\prime} \in F_{\lambda^{\prime}}} \gamma\left(G^{Q_{\lambda^{\prime}}^{i}(\mathbf{W})}, A_{\mathcal{Y}^{\lambda^{\prime}}}^{Q^{i}(\mathbf{W})}\right) \\
& =a_{s}+b_{s+1} .
\end{aligned}
$$

(The last equality follows from ([6).) Note that, if $s>k$, then $a_{s}=b_{s}=0$ because any partition $\lambda$ with $l(\lambda)=s$ satisfies $|\lambda| \geq s>k$. Hence, by (7),

$$
b_{0}=a_{0}+a_{1}+\cdots+a_{k},
$$

and the result follows.

We now deduce Theorem 1.1. Let $k, m \in \mathbb{N}$, and let $W$ be a vector space over $\mathbb{F}_{q}$ of dimension $m$. Consider the quiver representation $Q^{(k)}=\left(E_{0}, E_{1}, \mathbf{U}, \boldsymbol{\alpha}\right)$ given by (2): say, $E_{0}=\{a, b, c\}, U_{a}=V, U_{b}=Z, U_{c}=W(\operatorname{dim} V=k)$. Put $G=\mathrm{GL}(W)$ and $A=\mathcal{Y} \cap \operatorname{End}(W)$. Then, for any partition $\lambda \in \mathcal{P}^{m}$, the set $F_{\lambda}$ consists of just one flag, so

$$
\xi_{\lambda}(G, A)=\kappa_{\lambda}^{m}(\mathcal{Y}) .
$$

Moreover, $\mathbf{X}=\left(X_{a}, X_{b}, X_{c}\right) \mapsto X_{b}$ induces a one-to-one correspondence between the $G^{(k)}$-orbits in $A^{(k)}$ and the $\mathscr{P}(Z ; V)$-orbits in $\mathcal{Y} \cap \operatorname{End}(Z ; V)$. (Here we identify $V$ with its image under the injective map $V \rightarrow Z$.) Hence,

$$
\gamma\left(G^{(k)}, A_{\mathcal{Y}}^{(k)}\right)=\kappa_{(k)}^{k+m}(\mathcal{Y}) .
$$

Theorem 1.1 follows immediately from (8), (9) and Theorem 6.2.

Let $\lambda$ be a partition. It can be represented as

$$
\lambda=(\underbrace{s_{1}, s_{1}, \ldots, s_{1}}_{u_{1}}, \underbrace{s_{2}, \ldots, s_{2}}_{u_{2}}, \ldots, \underbrace{s_{l}, \ldots, s_{l}}_{u_{l}})
$$

where $s_{1}>s_{2}>\ldots>s_{l}$. Let $\bar{\lambda}$ be the set $\left\{s_{1}, \ldots, s_{l}\right\}$. If $S \subset \mathbb{N}$ is a finite set and $k \in \mathbb{N}$, let $r(k, S)$ be the number of partitions $\lambda$ such that $\bar{\lambda}=S$. That is, $r(k, S)$ is the number of partitions $\lambda$ such that $\lambda_{i} \in S$ for all $i$ and, for each $s \in S$, there exists $i$ such that $\lambda_{i}=s$.

If $S=\left\{s_{1}, \ldots, s_{l}\right\}$ is a set and $s_{1}>\cdots>s_{l}$, we shall identify the set $S$ with the partition $\left(s_{1}, s_{2}, \ldots, s_{l}\right)$. (So the notation $\xi_{S}(G, A)$ makes sense, for example.)

Let $W, G \leq \mathrm{GL}(W)$ and $A \subseteq \operatorname{End}(W) \cap \mathcal{Y}$ be as above, with $m=\operatorname{dim} W$. It is clear that, if $\lambda \in \mathcal{P}^{m}$, then

$$
\xi_{\lambda}(G, A)=\xi_{\bar{\lambda}}(G, A) .
$$


(Indeed, duplicating subspaces in a flag $\mathbf{W}$ does not add anything new to the structure.) Moreover, if a $S=S^{\prime} \sqcup\{m\}$ is a subset of $[1, m]$ containing $m$, then

$$
\xi_{S}(G, A)=\xi_{S^{\prime}}(G, A) .
$$

These identities allow us to simplify the expression in Theorem 6.2

Corollary 6.3. Under the hypotheses of Theorem 6.2,

$$
\gamma\left(G^{(k)}, A_{\mathcal{Y}}^{(k)}\right)=\sum_{j=0}^{k} c(k-j, \mathcal{Y}) \sum_{S \subseteq[1, m-1]}(r(j, S)+r(j, S \cup\{m\})) \xi_{S}(G, A) .
$$

Remark. When calculating the last sum, we only need to consider those $S \subseteq$ $[1, m-1]$ for which $\sum_{s \in S} s \leq k$ : if $\sum_{s \in S} s>k$, then $r(j, S)=r(j, S \cup\{m\})=0$ for all $j \in[0, k]$.

Similarly, Theorem 1.1 implies the following.

Corollary 6.4. Let $k, m \in \mathbb{N}$. Then

$$
\kappa_{(k)}^{k+m}(\mathcal{Y})=\sum_{j=0}^{k} c(k-j, \mathcal{Y}) \sum_{S \subseteq[1, m-1]}(r(k, S)+r(k, S \cup\{m\})) \kappa_{S}^{m}(\mathcal{Y}) .
$$

\section{Inverting the formula}

From now on, we shall assume that $\mathcal{Y}$ is the class $\mathcal{N}$ of all nilpotent endomorphisms, so $c(j, \mathcal{Y})=p(j)$ for all $j$. Let $W$ be an $m$-dimensional vector space over $\mathbb{F}_{q}$, where $m \in \mathbb{N}$ is fixed throughout the section. Let $G$ be a subgroup of $\mathrm{GL}(W)$ preserving a subset $A$ of $N(W)$ (as in Section $\left[\right.$ ). If $k \in \mathbb{Z}_{\geq 0}$, define

$$
\begin{aligned}
\phi_{k}(G, A) & =\sum_{S \subseteq[1, m]} r(k, S) \xi_{S}(G, A) \quad \text { and } \\
\psi_{k}(G, A) & =\phi_{k}(G, A)-\phi_{k-m}(G, A)
\end{aligned}
$$

where, by convention, $\phi_{k}(G, A)=0$ if $k<0$. If $S \subseteq[1, m-1]$, then

$$
r(k, S \cup\{m\})=r(k-m, S)+r(k-m, S \cup\{m\})
$$

for all $k \in \mathbb{N}$. Also, by (11), $\xi_{S}(G, A)=\xi_{S \cup\{m\}}(G, A)$. Hence,

$$
\begin{aligned}
\phi_{k-m}(G, A) & =\sum_{S \subseteq[1, m-1]} r(k-m, S) \xi_{S}(G, A) \\
& +\sum_{S \subseteq[1, m-1]} r(k-m, S \cup\{m\}) \xi_{S \cup\{m\}}(G, A) \\
& =\sum_{S \subseteq[1, m-1]} r(k, S \cup\{m\}) \xi_{S \cup\{m\}}(G, A) .
\end{aligned}
$$

It follows that

$$
\psi_{k}(G, A)=\sum_{S \subseteq[1, m-1]} r(k, S) \xi_{S}(G, A) .
$$

We now use Corollary 6.3 show that the numbers $\phi_{k}(G, A)$ and $\psi_{k}(G, A)$ may be expressed (independently of $G$ and $A$ ) in terms of $\gamma\left(G^{(r)}, A_{\mathcal{N}}^{(r)}\right), r \in[0, k]$. 
Lemma 7.1. Let $k \in \mathbb{N}$. There exist integers $a_{0}, a_{1}, \ldots, a_{k}$ that depend only on $k$ and $m$ (but not on $G$ or $A$ ) such that

$$
\phi_{k}(G, A)=\sum_{j=0}^{k} a_{j} \gamma\left(G^{(j)}, A_{\mathcal{N}}^{(j)}\right) .
$$

Proof. We prove the lemma by induction on $k$. Observe that

$$
\phi_{0}(G, A)=\xi_{\varnothing}(G, A)=\gamma\left(G^{(0)}, A_{\mathcal{N}}^{(0)}\right)=\gamma(G, A) .
$$

By Corollary 6.3

$$
\begin{aligned}
\gamma\left(G^{(k)}, A_{\mathcal{N}}^{(k)}\right) & =\sum_{j=0}^{k} p(k-j) \phi_{j}(G, A), \quad \text { so } \\
\phi_{k}(G, A) & =\gamma\left(G^{(k)}, A_{\mathcal{N}}^{(k)}\right)-p(k) \gamma(G, A)-\sum_{j=1}^{k-1} p(k-j) \phi_{j}(G, A) .
\end{aligned}
$$

The result for $\phi_{k}(G, A)$ now follows by the inductive hypothesis.

Corollary 7.2. Let $k \in \mathbb{N}$. There exist integers $a_{0}, a_{1}, \ldots, a_{k}$ that depend only on $k$ and $m$ (but not on $G$ or $A$ ) such that

$$
\psi_{k}(G, A)=\sum_{j=0}^{k} a_{j} \gamma\left(G^{(j)}, A_{\mathcal{N}}^{(j)}\right) .
$$

Also, there exist integers $a_{0}^{\prime}, a_{1}^{\prime}, \ldots, a_{k}^{\prime}$ depending only on $k$ and $m$ such that

$$
\gamma\left(G^{(k)}, A_{\mathcal{N}}^{(k)}\right)=\sum_{j=0}^{k} a_{j}^{\prime} \psi_{j}(G, A) .
$$

Proof. The first statement follows from Lemma [7.1. The second statement follows from (13) and the identity

$$
\phi_{j}(G, A)=\sum_{i=0}^{\infty} \psi_{j-i m}(G, A)
$$

which is a consequence of the definition of $\psi_{k}(G, A)$.

Let $n=m(m-1) / 2$. It is proved in the Appendix that there exist (explicitly defined) integers $c_{1}, \ldots, c_{n}$, depending only on $m$, such that

$$
r(k, S)=-\sum_{j=k-n}^{k-1} c_{k-j} r(j, S) .
$$

for all $k>n$ and all $S \subseteq[1, m-1]$.

Proposition 7.3. Let $n=m(m-1) / 2$. Let $k>n$. Then there exist integers $a_{k 0}, a_{k 1}, \ldots, a_{k n}$ depending only on $k$ and $m$ (but not on $G$ or $A$ ) such that

$$
\gamma\left(G^{(k)}, A_{\mathcal{N}}^{(k)}\right)=\sum_{j=0}^{n} a_{k j} \gamma\left(G^{(j)}, A_{\mathcal{N}}^{(j)}\right) .
$$


Proof. By (12) and (14), for all $k>n$,

$$
\psi_{k}(G, A)=-\sum_{j=k-n}^{k-1} c_{k-j} \psi_{j}(G, A) .
$$

An induction on $k$ shows that, for $k>n$, there exist integers $a_{k 1}^{\prime}, \ldots, a_{k n}^{\prime}$ such that

$$
\psi_{k}(G, A)=\sum_{j=1}^{n} a_{k j}^{\prime} \psi_{j}(G, A)
$$

By Corollary 7.2, the result follows.

Remark. The matrix formed by the numbers $r(k, S)$ as $k$ runs through $\mathbb{N}$ and $S$ runs through non-empty subsets of $[1, m-1]$ is of rank $m(m-1) / 2=n$, as shown in the Appendix. Thus, Proposition 7.3 is the best result that may be obtained via the method described.

We now show that Theorem 1.3 is a particular case of this result. Let $\mathbf{l}=\left(l_{1}, \ldots, l_{s}\right)$ be a tuple of nonnegative integers with $l_{1}+\cdots+l_{s}=m$. As before, let $W=\mathbb{F}_{q}^{m}$. Put $G=P^{\mathrm{l}}(q) \leq \mathrm{GL}(W)$ and $A=N^{\mathrm{l}}(q) \subseteq \operatorname{End}(W)$. Then $A$ is preserved by $G$. The quiver representation $Q^{k}$ may be depicted as

$$
U_{a} \longleftrightarrow U_{b} \longrightarrow W=U_{c}
$$

where the sequence is exact. Then the map $\operatorname{End}\left(Q^{k}\right) \rightarrow \operatorname{End}\left(U_{b}\right), \mathbf{X} \mapsto X_{b}$ establishes an isomorphism between the $G^{(k)}$-action on $A_{\mathcal{N}}^{(k)}$ and the $P^{k, 1}(q)$ action on $N^{k, 1}(q)$. (Here we identify $P^{k, 1}(q)$ with the subgroup of $\operatorname{GL}\left(U_{b}\right)$ consisting of those maps $g$ that preserve the image of $U_{a}$ and act as an element of $G$ on $W$.) Thus,

$$
\rho_{k, \mathbf{l}}(q)=\gamma\left(G^{(k)}, A_{\mathcal{N}}^{(k)}\right) .
$$

Theorem 1.3 now follows from Proposition 7.3 .

With $n=m(m-1) / 2$, as before, it is shown in the Appendix that there exist (explicitly defined) integers $d_{1}, \ldots, d_{n}$ such that

$$
\sum_{j=1}^{n} d_{j} r(j, S)= \begin{cases}1 & \text { if } S=[1, m-1], \\ 0 & \text { if } S \subsetneq[1, m-1] .\end{cases}
$$

Proposition 7.4. Let $m \in \mathbb{N}$, and let $W=\mathbb{F}_{q}^{m}$. Let $n=m(m-1) / 2$. Then there exist integers $a_{0}, a_{1}, \ldots, a_{n}$ depending only on $m$ such that, for any $G \leq$ $\mathrm{GL}(W)$ and $A \subseteq \mathrm{N}(W)$ with $G$ preserving $A$,

$$
\xi_{[1, m-1]}(G, A)=\sum_{j=0}^{n} a_{j} \gamma\left(G^{(j)}, A_{\mathcal{N}}^{(j)}\right) .
$$

Proof. By (16) and (12),

$$
\sum_{j=1}^{n} d_{j} \psi_{j}(G, A)=\xi_{[1, m-1]}(G, A) .
$$

The result now follows from Corollary 7.2 ,

In order to deduce Theorem 1.2, we put $G=\operatorname{GL}_{m}(q)$ and $A=\mathrm{N}_{m}(q)$. Then, by (15), $\rho_{(j, m)}(q)=\gamma\left(G^{(j)}, A^{(j)}\right)$. Also, $\xi_{[1, m-1]}(G, A)=\rho_{\left(1^{m}\right)}(q)$. Thus, Theorem 1.2 follows from Proposition [7.4. 


\section{Groups associated to preordered sets}

Let $C$ be a finite set. A binary relation $\preccurlyeq$ on $C$ is a preorder if

(i) $x \preccurlyeq x$ for all $x \in C$; and

(ii) if $x \preccurlyeq y$ and $y \preccurlyeq z$, then $x \preccurlyeq z$, for all $x, y, z \in C$.

A preordered set is a finite set together with a preorder on it. Two elements $x, y \in C$ are said to be comparable if either $x \preccurlyeq y$ or $y \preccurlyeq x$. If $(C, \preccurlyeq)$ is a preordered set, one can define an equivalence relation on $C$ as follows: $x$ is equivalent to $y$ if and only if $x \preccurlyeq y$ and $y \preccurlyeq x$. The clots of $C$ are, by definition, the equivalence classes with respect to this relation. Say that a clot $D \subseteq C$ is minimal if there is no $x \in C \backslash D$ such that $x \preccurlyeq y$ for some $y \in D$. A partially ordered set is a preordered set each of whose clots contains only one element. The $d u a l$ of a preordered set $(C, \preccurlyeq)$ is $\left(C, \preccurlyeq^{\prime}\right)$ where $x \preccurlyeq^{\prime} y$ if and only if $y \preccurlyeq x$. We denote the dual of a preordered set $C$ by $C^{*}$. If $\left(C_{1}, \preccurlyeq 1\right)$ and $\left(C_{2}, \preccurlyeq_{2}\right)$ are two preordered sets, their disjoint union is the set $C_{1} \sqcup C_{2}$ with the preorder $\preccurlyeq$ defined as follows: $x \preccurlyeq y$ if and only if $x, y \in C_{i}$ and $x \preccurlyeq_{i} y$ for some $i \in\{1,2\}$. Where appropriate, $C_{1} \sqcup C_{2}$ will denote the corresponding preordered set rather than just a set.

We shall define preordered sets by diagrams as follows. Clots will correspond to nodes, with the number at each node equal to the number of elements in the corresponding clot; $x \preccurlyeq y$ if and only if one can get from the node of $x$ to the node of $y$ by going along arrows. For example,

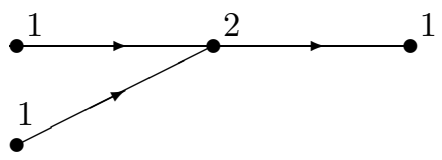

is (isomorphic to) the preordered set $\{1,2,3,4,5\}$ where $\preccurlyeq$ is defined as follows: $1 \preccurlyeq 3 \preccurlyeq 4 \preccurlyeq 3 \preccurlyeq 5$ and $2 \preccurlyeq 3$.

Let $q$ be a prime power. Suppose $(C, \preccurlyeq)$ is a (finite) preordered set. Let $M^{C}(q)=M^{(C, \preccurlyeq)}(q)$ be the set of all $C \times C$ matrices $X=\left(x_{i j}\right)$ over $\mathbb{F}_{q}$ such that $x_{i j}=0$ unless $i \preccurlyeq j$, for all $i, j \in C$. Let $P^{C}(q)$ be the group of all invertible matrices in $M^{C}(q)$, and let $N^{C}(q)$ be the set of all nilpotent matrices in $M^{C}(q)$. Let

$$
\rho_{C}(q)=\gamma\left(P^{C}(q), N^{C}(q)\right) .
$$

For example, if $C$ is the preordered set given by (17), then $M^{C}(q)$ is (up to a permutation of $C$ ) the set of matrices of the form

$$
\left(\begin{array}{lllll}
* & 0 & * & * & * \\
0 & * & * & * & * \\
0 & 0 & * & * & * \\
0 & 0 & * & * & * \\
0 & 0 & 0 & 0 & *
\end{array}\right) .
$$

If $\mathbf{l}=\left(l_{1}, \ldots, l_{s}\right)$ is a tuple of nonnegative integers, we shall also write $\mathbf{l}$ for the preordered set

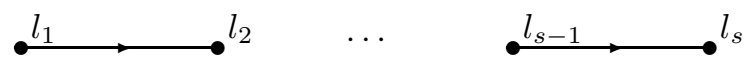

Note that then $P^{\mathbf{l}}(q), \rho_{\mathbf{l}}(q)$, etc. are as defined previously.

If $C$ is a preordered set, we shall aim to reduce the problem of finding $\rho_{C}(q)$ to orbit-counting problems for matrices of size less than $|C|$, using Theorem 6.2 In some cases, we shall be able to express $\rho_{C}(q)$ in terms of $\rho_{O}(q)$ where $O$ varies 
among preordered sets of size less than $|C|$. This will allow us to calculate $\rho_{C}(q)$ using recursion for some $C$.

Let $(C, \preccurlyeq)$ be a finite preordered set. Let $D \subseteq C$ be a minimal clot in $C$ with $|D|=k$, say. Let $E=\{x \in C: y \npreceq x \forall y \in D\}$. Let $\bar{D}=C \backslash D, \bar{E}=C \backslash E$ and $C^{\prime}=C \backslash(D \cup E)$. We shall view $C \times C$ matrices as endomorphisms of a vector space $V$ over $\mathbb{F}_{q}$ equipped with a basis $\left\{e_{i}\right\}_{i \in C}$. Let $V_{D}=\operatorname{span}\left\{e_{i}\right\}_{i \in D}$, $V_{E}=\operatorname{span}\left\{e_{i}\right\}_{i \in E}, V_{\bar{D}}=V / V_{D}$ and $V_{C^{\prime}}=V /\left(V_{D}+V_{E}\right)$. We may view $M^{\bar{D}}(q)$ as a subring of $\operatorname{End}\left(V_{\bar{D}}\right)$ using the projections of $e_{i}, i \in \bar{D}$ as a basis of $V_{\bar{D}}$. Similarly, we may view $M^{C^{\prime}}(q)$ as a subring of $\operatorname{End}\left(V_{C^{\prime}}\right)$. (By abuse of notation, we shall view $\left\{e_{i}\right\}_{i \in C^{\prime}}$ as a basis of $V_{C^{\prime}}$.) Let $m=\left|C^{\prime}\right|$. If

$$
S=\left\{s_{1}>\cdots>s_{r}\right\} \subseteq[1, m-1],
$$

let $\mathcal{H}_{S}=\mathcal{H}_{S}\left(C^{\prime}\right)$ be the set of all flags $\mathbf{W}=\left(W_{1}, \ldots, W_{r}\right)$ such that

$$
W_{r} \leq W_{r-1} \leq \cdots \leq W_{1} \leq V_{C^{\prime}}
$$

and $\operatorname{dim} W_{i}=s_{i}$ for all $i$. Let $\pi: V_{\bar{D}} \rightarrow V_{C^{\prime}}$ be the natural projection. If $\mathbf{W} \in \mathcal{H}_{S}$, write $\pi^{-1}(\mathbf{W})$ for the flag $\left(\pi^{-1}\left(W_{1}\right), \ldots, \pi^{-1}\left(W_{r}\right)\right)$ in $V_{\bar{D}}$. Let $M_{\mathbf{W}}^{\bar{D}}(q)=\mathscr{P}\left(V_{\bar{D}} ; \pi^{-1}(\mathbf{W})\right)$. That is, $M_{\mathbf{W}}^{\bar{D}}(q)$ is the ring of all elements of $M^{D}(q)$ whose action on $V_{C^{\prime}}$ preserves $\mathbf{W}$. Let $P_{\mathbf{W}}^{\bar{D}}(q)$ be the group of all invertible elements in $M_{\mathbf{W}}^{\bar{D}}(q)$, and let $N_{\mathbf{W}}^{\bar{D}}(q)$ be the set of all nilpotent elements in $M_{\mathbf{W}}^{\bar{D}}(q)$.

Proposition 8.1. With the notation as in the previous paragraph, suppose that $H_{S}$ is a complete set of representatives of the $P^{C^{\prime}}(q)$-orbits on $\mathcal{H}_{S}$ for each $S \subseteq[1, m-1]$. Then

$\rho_{C}(q)=\sum_{j=0}^{k} p(k-j) \sum_{S \subseteq[1, m-1]}(r(j, S)+r(j, S \cup\{m\})) \sum_{\mathbf{W} \in H_{S}} \gamma\left(P_{\mathbf{W}}^{\bar{D}}(q), N_{\mathbf{W}}^{\bar{D}}(q)\right)$.

Proof. Let $Z$ be a complete set of representatives of $P^{\bar{D}}(q)$-orbits on $N^{\bar{D}}(q)$. Let $\pi_{D}: M^{C}(q) \rightarrow M^{\bar{D}}(q)$ be the natural projection. If $X \in Z$, let $G_{X}=$ $\pi_{D}^{-1}\left(\operatorname{Stab}_{P^{\bar{D}}(q)}(X)\right) \cap P^{C}(q)$ and $A_{X}=\pi_{D}^{-1}(X) \cap N^{C}(q)$. By Lemma 4.2,

$$
\rho_{C}(q)=\gamma\left(P^{C}(q), N^{C}(q)\right)=\sum_{X \in Z} \gamma\left(G_{X}, A_{X}\right) .
$$

Let $\pi_{E}: M^{C}(q) \rightarrow M^{\bar{E}}(q)$ be the natural projection. Let $X \in Z$. It is easy to see that $\pi_{E}$ establishes a bijection between the $G_{X}$-orbits on $A_{X}$ and the $\pi_{E}\left(G_{X}\right)$-orbits on $\pi_{E}\left(A_{X}\right)$. So

$$
\gamma\left(G_{X}, A_{X}\right)=\gamma\left(\pi_{E}\left(G_{X}\right), \pi_{E}\left(A_{X}\right)\right)
$$

Let $Q$ be the quiver representation

$$
V_{D} \longrightarrow V_{\bar{E}} \longrightarrow V_{C^{\prime}}
$$

where the first map is induced by the inclusion $V_{D} \hookrightarrow V_{C}$ and the second map is the natural projection. Then $Q$ is isomorphic to $Q^{k}$ in the notation of Section 6 $\left(V_{C^{\prime}}\right.$ plays the role of $\left.W\right)$. Let $\chi: \operatorname{End}(Q) \rightarrow \operatorname{End}\left(V_{\bar{E}} ; V_{D}\right)$ be the isomorphism which maps an endomorphism $\mathbf{Y}$ of $Q$ to the action of $\mathbf{Y}$ on $V_{\bar{E}}$.

Our aim is to express $\gamma\left(\pi_{E}\left(G_{X}\right), \pi_{E}\left(A_{X}\right)\right)$ by applying Corollary 6.3 to the quiver $Q^{k}$. In fact, this expression is the only substantial step of the proof. 
Let $G_{X}^{\prime} \leq P^{C^{\prime}}(q)$ be the image of $G_{X}$ under the natural map $P^{C}(q) \rightarrow$ $P^{C^{\prime}}(q)$. Let $\eta: M^{\bar{D}}(q) \rightarrow M^{C^{\prime}}(q)$ be the natural projection, and let $A_{X}^{\prime}=$ $\{\eta(X)\} \subseteq N^{C^{\prime}}(q)$. Let $\omega: \operatorname{End}\left(V_{\bar{E}} ; V_{D}\right) \rightarrow \mathrm{M}_{C^{\prime}, C^{\prime}}(q)$ be the natural projection, $\left(y_{i j}\right)_{i, j \in \bar{E}} \mapsto\left(y_{i j}\right)_{i, j \in C^{\prime}}$. Since $d \preccurlyeq c$ for all $d \in D$ and $c \in C^{\prime}$,

$$
\omega^{-1}\left(M^{C^{\prime}}(q)\right)=M^{\bar{E}}(q) .
$$

We identify the quiver representation $Q$ with $Q^{k}$; let $\left(G_{X}^{\prime}\right)^{(k)}$ and $\left(A_{X}^{\prime}\right)_{\mathcal{N}}^{(k)}$ be as defined in Section 6. Then, by those definitions,

$$
\begin{aligned}
& \chi\left(\left(G_{X}^{\prime}\right)^{(k)}\right)=\omega^{-1}\left(G_{X}^{\prime}\right) \cap P^{\bar{E}}(q) \text { and } \\
& \chi\left(\left(A_{X}^{\prime}\right)_{\mathcal{N}}^{(k)}\right)=\omega^{-1}\left(A_{X}^{\prime}\right) \cap N^{\bar{E}}(q) .
\end{aligned}
$$

Hence, clearly, $\chi\left(\left(A_{X}^{\prime}\right)_{\mathcal{N}}^{(k)}\right)=\pi_{E}\left(A_{X}\right)$.

We claim that $\chi\left(\left(G_{X}^{\prime}\right)^{(k)}\right)=\pi_{E}\left(G_{X}\right)$. It is clear that $\pi_{E}\left(G_{X}\right) \subseteq \omega^{-1}\left(G_{X}^{\prime}\right) \cap$ $P^{\bar{E}}(q)$. For the converse, suppose that $Y=\left(y_{i j}\right) \in P^{\bar{E}}(q)$ satisfies $\omega(Y) \in G_{X}^{\prime}$. Then there exists a matrix $B=\left(b_{i j}\right) \in M^{C}(q)$ such that $\pi_{D}(B)$ fixes $X$ and $b_{i j}=y_{i j}$ for all $i, j \in C^{\prime}$. Define $R=\left(r_{i j}\right) \in M^{C}(q)$ by 'gluing together' $\pi_{D}(B)$ and $Y: r_{i j}=y_{i j}$ if $i, j \in D \cup C^{\prime}$ and $r_{i j}=b_{i j}$ if $i, j \in E \cup C^{\prime}$. Then $\pi_{D}(R)=\pi_{D}(B)$, so $R \in G_{X}$, and $\pi_{E}(R)=Y$. Hence, $Y \in \pi_{E}\left(G_{X}\right)$.

We are now in a position to apply Corollary 6.3.

$$
\begin{aligned}
& \gamma\left(\pi_{E}\left(G_{X}\right), \pi_{E}\left(A_{X}\right)\right)=\gamma\left(\left(G_{X}^{\prime}\right)^{(k)},\left(A_{X}^{\prime}\right)_{\mathcal{N}}^{(k)}\right) \\
& \quad=\sum_{j=0}^{k} p(k-j) \sum_{S \subseteq[1, m-1]}(r(j, S)+r(j, S \cap\{m\})) \xi_{S}\left(G_{X}^{\prime}, A_{X}^{\prime}\right)
\end{aligned}
$$

By definition,

$$
\begin{aligned}
\xi_{S}\left(G_{X}^{\prime}, A_{X}^{\prime}\right) & =\gamma\left(G_{X}^{\prime},\left\{\mathbf{W} \in \mathcal{H}_{S}: X\left(\pi^{-1}(\mathbf{W})\right)=\pi^{-1}(\mathbf{W})\right\}\right) \\
& =\gamma\left(\operatorname{Stab}_{P} \overline{\bar{D}}(q)\right. \\
& \left.(X),\left\{\mathbf{W} \in \mathcal{H}_{S}: X\left(\pi^{-1}(\mathbf{W})\right)=\pi^{-1}(\mathbf{W})\right\}\right) .
\end{aligned}
$$

Let

$$
L=\left\{(X, \mathbf{W}) \in N^{\bar{D}}(q) \times \mathcal{H}_{S}: X\left(\pi^{-1}(\mathbf{W})\right)=\pi^{-1}(\mathbf{W})\right\}
$$

By Lemma 4.2 (applied in two different ways),

$$
\sum_{X \in Z} \xi_{S}\left(G_{X}^{\prime}, A_{X}^{\prime}\right)=\gamma\left(P^{\bar{D}}(q), L\right)=\sum_{\mathbf{W} \in H_{S}} \gamma\left(P_{\mathbf{W}}^{\bar{D}}(q), N_{\mathbf{W}}^{\bar{D}}(q)\right) .
$$

The result follows by combining (18), (19), (20) and (21).

Therefore, in order to compute $\rho_{C}(q)$, it is enough to find a set of representatives $H_{S}$ for each $S \subseteq[1, m-1]$ and to calculate $\gamma\left(P_{\mathbf{W}}^{\bar{D}}(q), N_{\mathbf{W}}^{\bar{D}}(q)\right)$ for every $\mathbf{W} \in H_{S}$.

Suppose that any two elements of $C^{\prime}$ are comparable. Then $C^{\prime}$ is isomorphic to some $\mathbf{l}=\left(l_{1}, \ldots, l_{s}\right)$. Let $S=\left\{t_{1}>\cdots>t_{u}\right\} \subseteq[1, m-1]$. The orbits of $P^{\mathbf{l}}(q)$ on $\mathcal{H}_{S}(\mathbf{l})$ are well understood. (In fact, these orbits correspond to orbits of pairs of flags, of appropriate dimensions, under the action of $\mathrm{GL}_{m}(q)$.) Let $\mathcal{A}_{S}^{1}$ be the set of tuples $\mathbf{n}=\left(n_{i j}\right)_{i \in[1, s], j \in[1, u]}$ of nonnegative integers satisfying

$$
\begin{gathered}
l_{i} \geq n_{i 1} \geq n_{i 2} \geq \cdots \geq n_{i u} \quad \text { for all } i \in[1, s] \text { and } \\
\sum_{i=1}^{s} n_{i j}=t_{j} \quad \text { for all } j \in[1, u] .
\end{gathered}
$$


Relabel the basis $\left\{e_{i}\right\}_{i \in C^{\prime}}$ of $V_{C^{\prime}}$ as $\left\{f_{i j}\right\}_{i \in[1, s], j \in\left[1, l_{i}\right]}$ so that $P^{\mathrm{l}}(q)=P^{C^{\prime}}(q)$ is the stabiliser of the flag consisting of the subspaces $\operatorname{span}\left\{f_{i j}\right\}_{i \in[1, r], j \in\left[1, l_{i}\right]}$, $r=1, \ldots, s$. (This relabelling is given by an isomorphism between $C^{\prime}$ and l.) For each $\mathbf{n} \in \mathcal{A}_{S}^{\mathbf{l}}$, define a flag $\mathbf{W}^{\mathbf{n}} \in \mathcal{H}_{S}(\mathbf{l})$ as follows:

$$
W_{a}^{\mathbf{n}}=\operatorname{span}\left\{f_{i j}: 1 \leq i \leq s, 1 \leq j \leq n_{i a}\right\}, \quad a=1,2, \ldots u .
$$

Lemma 8.2. [5, Example 2.10] Let $\mathbf{l}=\left(l_{1}, \ldots, l_{s}\right)$ be a tuple of nonnegative integers with $m=l_{1}+\cdots+l_{s}$. Let $S \subseteq[1, m-1]$. Then $\left\{\mathbf{W}^{\mathbf{n}}: \mathbf{n} \in \mathcal{A}_{S}^{1}\right\}$ is a complete set of representatives of $P^{\mathbf{l}}(q)$-orbits on $\mathcal{H}_{S}(\mathbf{l})$.

If $C^{\prime}$ is isomorphic to some $\mathbf{l}$, we choose a set of representatives $H_{S}$ as given by Lemma 8.2. In particular, for every $\mathbf{W} \in H_{S}$, each $W_{j}$ is spanned by a subset of the standard basis $\left\{e_{i}\right\}_{i \in C^{\prime}}$.

Let $\mathbf{n} \in \mathcal{A}_{S}^{1}$. Write $n_{i 0}=l_{i}$ and $n_{i, u+1}=0$ for all $i \in[1, s]$. Define a new preorder $\preccurlyeq^{\prime}$ on $\bar{D}$ as follows:

(i) suppose $i_{1}, i_{2} \in C^{\prime}$; let $a_{1}, b_{1}, a_{2}, b_{2}$ be such that $e_{i_{1}}=f_{a_{1}, b_{1}}$ and $e_{j}=$ $f_{a_{2}, b_{2}}$; then $i_{1} \preccurlyeq^{\prime} i_{2}$ if and only if $a_{1} \leq a_{2}$ and there exists $c \in[0, u]$ such that $b_{1} \leq n_{a_{1}, c}$ and $b_{2}>n_{a_{2}, c+1}$;

(ii) if either $i \notin C^{\prime}$ or $j \notin C^{\prime}$, then $i \preccurlyeq{ }^{\prime} j$ if and only if $i \preccurlyeq j$.

Note that, in case (ii), $i_{1} \preccurlyeq i_{2}$ if and only if $a_{1} \leq a_{2}$. It is straightforward to check that, if $i_{1}, i_{2} \in C^{\prime}$, then $i_{1} \preccurlyeq^{\prime} i_{2}$ if and only if $i_{1} \preccurlyeq i_{2}$ and, for all $j \in[1, u]$, $e_{i_{1}} \in W_{j}^{\mathbf{n}}$ implies $e_{i_{2}} \in W_{j}^{\mathbf{n}}$. (In fact, this is our motivation for defining $\preccurlyeq^{\prime}$ ).

Let $O=O(S, \mathbf{n})$ be the preordered set $\left(\bar{D}, \preccurlyeq^{\prime}\right)$. It follows that $M_{\mathbf{W}^{\mathbf{n}}}^{\bar{D}}(q)=$ $M^{O(S, \mathbf{n})}(q)$. Hence,

$$
\gamma\left(P_{\mathbf{W}^{\mathbf{n}}}^{\bar{D}}(q), N_{\mathbf{W}^{\mathbf{n}}}^{\bar{D}}(q)\right)=\rho_{O(S, \mathbf{n})}(q),
$$

and we deduce the following from Proposition 8.1

Corollary 8.3. Let $(C, \preccurlyeq)$ be a preordered set with a minimal clot D. Let $k=|D|$. Let $C^{\prime}=\{x \in C: y \preccurlyeq x \forall y \in D\} \backslash D$. Let $m=\left|C^{\prime}\right|$. Suppose the preordered set $C^{\prime}$ is isomorphic to some $\mathbf{l}=\left(l_{1}, \ldots, l_{s}\right)$. Then

$$
\rho_{C}(q)=\sum_{j=0}^{k} p(k-j) \sum_{S \subseteq[1, m-1]}(r(j, S)+r(j, S \cup\{m\})) \sum_{\mathbf{n} \in \mathcal{A}_{S}^{1}} \rho_{O(S, \mathbf{n})}(q),
$$

where $O(S, \mathbf{n})$ is defined in terms of $\bar{D}=C \backslash D, C^{\prime}$ and $\preccurlyeq$ as above.

Since $|\bar{D}|<|C|$, this allows us to compute $\rho_{C}(q)$ by recursion as long as we can always find a minimal clot $D$ such that any two elements of $C^{\prime}$ are comparable. Using this method, one may compute $\rho_{\left(l_{1}, \ldots, l_{s}\right)}(q)$ for all tuples $\mathbf{l}$ with $l_{1}+\cdots+l_{s} \leq 6$, thus proving Proposition 1.4 (the explicit expressions are given at the end of Section (9). Indeed, one can choose the clots $D$ in such a way that the only preordered set occurring in that computation for which Corollary 8.3 is not applicable is the one considered in Example 8.3 below. For more detail on the computation, see [4, Appendix B]. (The computation also uses the symmetry $\rho_{C}(q)=\rho_{C^{*}}(q)$ proved in Section 9 below.)

We now consider some examples of computations using Proposition 8.1 and Corollary 8.3. For ease of notation, we list the flags $\mathbf{W}=\mathbf{W}^{\mathbf{n}} \in H_{S}$ directly, without giving $\mathbf{n}$.

Example 8.1. Suppose $C$ is isomorphic to $(1,1,1,1)$ : say, $C=[1,4]$ with the usual order. The only minimal clot is $D=\{1\}$. Then $E=\varnothing$ and $C^{\prime}=\{2,3,4\}$. 
The only non-zero terms in Corollary 8.3 are given by $S=\varnothing$ and $S=\{1\}$. If $S=\varnothing$, we get a summand $\rho_{\left(1^{3}\right)}(q)$. Assume $S=\{1\}$. Then any $\mathbf{W} \in \mathcal{H}_{S}$ consists of a single 1-dimensional space $W_{1}$. So $H_{S}$ consists of $\left\langle e_{2}\right\rangle,\left\langle e_{3}\right\rangle$ and $\left\langle e_{4}\right\rangle$. If $W_{1}=\left\langle e_{2}\right\rangle$, then $O \simeq\left(1^{3}\right)$, so we get another term $\rho_{\left(1^{3}\right)}(q)$. If $W_{1}=\left\langle e_{3}\right\rangle$, $O$ is isomorphic to the preordered set $\Delta_{1}$ given by the diagram

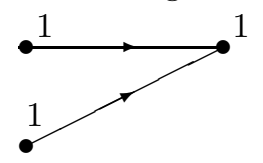

Finally, if $W_{1}=\left\langle e_{4}\right\rangle$, then $O \simeq(1,1) \sqcup(1)$, so we get a term $\rho_{(1,1)}(q)$. Hence,

$$
\rho_{\left(1^{4}\right)}(q)=2 \rho_{\left(1^{3}\right)}(q)+\rho_{\Delta_{1}}(q)+\rho_{\left(1^{2}\right)}(q) .
$$

Example 8.2. We compute $\rho_{\Delta_{1}}(q)$, where $\Delta_{1}$ is given by (22). We may take for $D$ either of the minimal elements of $\Delta_{1}$. Then $E$ consists of the other minimal element, and $C^{\prime}$ consists of the maximal element (label the elements 1,2,3 so that 3 is the maximal element). If $S=\varnothing$, we get $\rho_{\left(1^{2}\right)}(q)$. If $S=\{1\}$, then necessarily $W_{1}=\left\langle e_{3}\right\rangle$ and we get $\rho_{\left(1^{2}\right)}(q)$ again. Hence, $\rho_{\Delta_{1}}(q)=2 \rho_{\left(1^{2}\right)}(q)=4$ for all $q$.

Example 8.3. We now compute $\rho_{\Delta_{2}}(q)$ where $\Delta_{2}$ is the partially ordered set

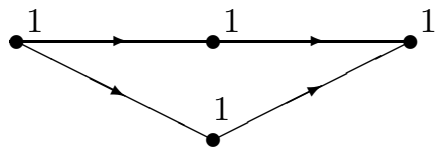

We may assume that the elements of $\Delta_{2}$ are $1,2,3,4$ where 1 is the smallest element and 4 is the largest. The only minimal clot is $D=\{1\}$. We have $C^{\prime}=$ $\{2,3,4\}$. In this case, $C^{\prime}$ is not isomorphic to any $\mathbf{l}$, so we apply Proposition 8.1 directly. If $S=\varnothing$, we get the term $\rho_{\Delta_{1}}(q)$. Assume $S=\{1\}$. The following is a complete set of representatives for the action of $P^{C^{\prime}}(q)$ on the 1-dimensional subspaces $W_{1}$ in $V_{C^{\prime}}$ :

$$
\left\{\left\langle e_{2}\right\rangle,\left\langle e_{3}\right\rangle,\left\langle e_{4}\right\rangle,\left\langle e_{2}+e_{3}\right\rangle\right\} .
$$

In each of the first two cases, $\gamma\left(P_{\mathbf{W}}^{\bar{D}}(q), N_{\mathbf{W}}^{\bar{D}}(q)\right)=\rho_{\Delta_{1}}(q)$. If $W_{1}=\left\langle e_{4}\right\rangle$, then $N_{\mathbf{W}}^{\bar{D}}(q)$ contains just the zero matrix, so the corresponding term in the sum is equal to 1 . Finally, suppose $W_{1}=\left\langle e_{2}+e_{3}\right\rangle$. Then we do not get a term of the form $\rho_{O}(q)$ for a preordered set $O$. It is possible to apply Corollary 5.3 to find the corresponding term, but in this case we may find the orbits directly. With respect to the basis $\left\{e_{2}, e_{3}, e_{4}\right\}$, we have:

$$
\begin{aligned}
& P_{\mathbf{W}}^{C^{\prime}}(q)=\left\{\left(\begin{array}{lll}
a & 0 & * \\
0 & a & * \\
0 & 0 & b
\end{array}\right): a, b \in \mathbb{F}_{q} \backslash\{0\}\right\} \text { and } \\
& N_{\mathbf{W}}^{C^{\prime}}(q)=\left\{\left(\begin{array}{lll}
0 & 0 & * \\
0 & 0 & * \\
0 & 0 & 0
\end{array}\right)\right\} .
\end{aligned}
$$

It is easy to see that a complete set of representatives of $P_{\mathbf{W}}^{C^{\prime}}(q)$-orbits on $N_{\mathbf{W}}^{C^{\prime}}(q)$ is

$$
\left\{\left(\begin{array}{lll}
0 & 0 & 0 \\
0 & 0 & 0 \\
0 & 0 & 0
\end{array}\right),\left(\begin{array}{lll}
0 & 0 & 1 \\
0 & 0 & 0 \\
0 & 0 & 0
\end{array}\right),\left(\begin{array}{lll}
0 & 0 & a \\
0 & 0 & 1 \\
0 & 0 & 0
\end{array}\right): a \in \mathbb{F}_{q}\right\} .
$$

So in this case $\gamma\left(P_{\mathbf{W}}^{C^{\prime}}(q), N_{\mathbf{W}}^{C^{\prime}}(q)\right)=q+2$. Thus,

$$
\rho_{\Delta_{2}}(q)=3 \rho_{\Delta_{1}}(q)+1+(q+2)=q+15 .
$$


Example 8.4. We consider $\rho_{(2,2,2)}(q)$. $(2,2,2)$ is isomorphic to $C=[1,6]$ with clots $\{1,2\},\{3,4\}$ and $\{5,6\}$ (in the increasing order). The only minimal clot is $D=\{1,2\}$, so $C^{\prime}=\{3,4,5,6\}$. The only non-zero terms in Corollary 8.3 come from $S=\varnothing, S=\{1\}$ and $S=\{2\}$. If $S=\varnothing$, we get $p(2) \rho_{(2,2)}=2 \rho_{(2,2)}$. If $S=\{1\}, H_{S}$ contains two flags $\mathbf{W}=\left(W_{1}\right)$, namely $W_{1}=\left\langle e_{3}\right\rangle$ and $W_{1}=\left\langle e_{5}\right\rangle$. In the first case, we get $\rho_{(1,1,2)}(q)$. In the second case, $O$ is isomorphic to the preordered set $\Delta_{3}$ given by

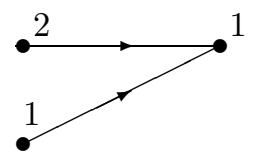

These two terms occur with the multiple 2 in the sum because $r(2,\{1\})=$ $r(1,\{1\})=1$. Finally, if $S=\{2\}$, the possibilities for $W_{1}$ are: $\left\langle e_{3}, e_{4}\right\rangle,\left\langle e_{3}, e_{5}\right\rangle$ and $\left\langle e_{5}, e_{6}\right\rangle$. These give rise to the terms $\rho_{(2,2)}(q), \rho_{\Delta_{2}}(q)$ and 4 respectively. (In the last case, $O \simeq(2) \sqcup(2)$.) Thus,

$$
\rho_{(2,2,2)}(q)=3 \rho_{(2,2)}(q)+2 \rho_{(1,1,2)}(q)+2 \rho_{\Delta_{3}}(q)+\rho_{\Delta_{2}}(q)+4 .
$$

\section{Dual quiver representations}

Let $Q=\left(E_{0}, E_{1}, \mathbf{U}, \boldsymbol{\alpha}\right)$ be a quiver representation over a field $\mathbb{F}_{q}$. Define the dual representation $Q^{*}=\left(E_{0}, E_{1}^{*}, \mathbf{U}^{*}, \boldsymbol{\alpha}^{*}\right)$ as follows:

(i) $E_{1}^{*}$ is obtained by inverting all the arrows in $E_{1}$ : for each $e \in E_{1}, \sigma^{*}(e)=$ $\tau(e)$ and $\tau^{*}(e)=\sigma(e)$;

(ii) $\mathbf{U}=\left(U_{a}^{*}\right)_{a \in E_{0}}$ where $U_{a}^{*}$ is the dual space to $U_{a}$;

(iii) $\boldsymbol{\alpha}^{*}=\left(\alpha_{e}^{*}\right)_{e \in E_{1}}$ where $\alpha_{e}^{*}$ is the dual map to $\alpha_{e}$.

Recall that $\theta(Q)=\gamma(\operatorname{Aut}(Q), N(Q))$. If $\mathbf{X} \in \operatorname{End}(Q)$, let $\mathbf{X}^{*} \in \operatorname{End}\left(Q^{*}\right)$ be the endomorphism $\left(X_{a}^{*}\right)_{a \in E_{0}}$.

Proposition 9.1. Let $Q=\left(E_{0}, E_{1}, \mathbf{U}, \boldsymbol{\alpha}\right)$ be a quiver representation. The map $\mathbf{X} \mapsto \mathbf{X}^{*}$ is a ring isomorphism between $\operatorname{End}(Q)$ and $\operatorname{End}\left(Q^{*}\right)^{\text {op }}$. Hence, $\theta(Q)=\theta\left(Q^{*}\right)$.

Proof. Clearly, $\mathbf{X} \mapsto \mathbf{X}^{*}$ is a ring homomorphism from $\operatorname{End}(Q)$ to $\operatorname{End}\left(Q^{*}\right)^{\text {op }}$. If $V$ is a finite dimensional vector space, $V^{* *}$ is naturally equivalent to $V$. This equivalence establishes an isomorphism from $Q^{* *}$ onto $Q$, which identifies $\mathbf{X}^{* *}$ and $\mathbf{X}$ for all $\mathbf{X} \in \operatorname{End}(Q)$. Hence, $\mathbf{X} \mapsto \mathbf{X}^{*}$ is a bijection.

Now let $(C, \preccurlyeq)$ be a preordered set. Let $V$ be a vector space over $\mathbb{F}_{q}$ with a basis $\left\{e_{i}\right\}_{i \in C}$. As in the previous section, we may then identify $M^{C}(q)$ with a subring of $\operatorname{End}(V)$ using this basis.

For each $i \in C$, let $\mathcal{D}(i)=\{j \in C: j \preccurlyeq i\}$. Let $T_{C}=T_{C}(q)$ be the quiver representation $\left(C \sqcup\{0\}, E_{1}, \mathbf{U}, \boldsymbol{\alpha}\right)$ such that

(i) $E_{1}$ is equal to $C$ as a set, $\sigma(i)=i$ and $\tau(i)=0$ for all $i \in C$;

(ii) $U_{0}=V$, and $U_{i}=\operatorname{span}\left\{e_{j}\right\}_{j \in \mathcal{D}(i)} \leq V$ for all $i \in C$;

(iii) $\alpha_{i}$ is the inclusion map $U_{i} \hookrightarrow V$ for each $i \in C$.

It is easy to check that $\mathbf{X} \mapsto X_{0}$ is a ring isomorphism from $\operatorname{End}\left(T_{C}\right)$ onto $M^{C}(q)$. Hence,

$$
\rho_{C}(q)=\theta\left(T_{C}(q)\right)
$$

The following is an easy exercise. 
Proposition 9.2. Let $C$ be a preordered set. Then the rings $\operatorname{End}\left(T_{C}^{*}\right)$ and $M^{C^{*}}(q)$ are isomorphic. Hence, $\rho_{C^{*}}(q)=\theta\left(T_{C}^{*}\right)$.

Combining (23), Proposition 9.1 and Proposition 9.2, we obtain the following result.

Corollary 9.3. For any finite preordered set $C$, for all prime powers $q$,

$$
\rho_{C}(q)=\rho_{C^{*}}(q) .
$$

In particular, if $\left(l_{1}, \ldots, l_{s}\right)$ is a tuple of nonnegative integers, then

$$
\rho_{\left(l_{1}, \ldots, l_{s}\right)}(q)=\rho_{\left(l_{s}, \ldots, l_{1}\right)}(q) .
$$

The following table gives the values of $\rho_{\mathbf{l}}(q)$ for all tuples $\mathbf{l}=\left(l_{1}, \ldots, l_{s}\right)$ with $l_{1}+\cdots+l_{s} \leq 6$ (see [4, Appendix B] for a detailed computation). In view of Corollary 9.3, we list only one representative for each pair of distinct tuples

\begin{tabular}{|c|c|}
\hline 1 & $\rho_{\mathbf{l}}(q)$ \\
\hline (1) & 1 \\
\hline$(2)$ & 2 \\
\hline$(1,1)$ & 2 \\
\hline (3) & 3 \\
\hline$(1,2)$ & 4 \\
\hline$(1,1,1)$ & 5 \\
\hline$(4)$ & 5 \\
\hline$(1,3)$ & 7 \\
\hline$(2,2)$ & 10 \\
\hline$(1,1,2)$ & 12 \\
\hline$(1,2,1)$ & 11 \\
\hline$(1,1,1,1)$ & 16 \\
\hline (5) & 7 \\
\hline$(1,4)$ & 12 \\
\hline $\begin{array}{l}(2,3) \\
\end{array}$ & 18 \\
\hline$(1,2,2)$ & 30 \\
\hline$(2,1,2)$ & 31 \\
\hline$(1,1,3)$ & 23 \\
\hline$(1,3,1)$ & 21 \\
\hline$(1,1,1,2)$ & 43 \\
\hline$(1,1,2,1)$ & 40 \\
\hline$(1,1,1,1,1)$ & 61 \\
\hline$(6)$ & 11 \\
\hline$(1,5)$ & 19 \\
\hline$(2,4)$ & 34 \\
\hline$(3,3)$ & 37 \\
\hline$(1,2,3)$ & 63 \\
\hline$(1,3,2)$ & 62 \\
\hline$(2,1,3)$ & 66 \\
\hline$(1,1,4)$ & 43 \\
\hline$(1,4,1)$ & 38 \\
\hline$(2,2,2)$ & $q+89$ \\
\hline$(1,1,1,3)$ & 93 \\
\hline
\end{tabular}
$\left(l_{1}, \ldots, l_{s}\right)$ and $\left(l_{s}, \ldots, l_{1}\right)$.

Continued on the next page 


\begin{tabular}{|c|c|}
\hline $\mathbf{l}$ & $\rho_{\mathbf{l}}(q)$ \\
\hline$(1,1,3,1)$ & 84 \\
\hline$(1,1,2,2)$ & $q+121$ \\
\hline$(1,2,1,2)$ & 120 \\
\hline$(1,2,2,1)$ & 113 \\
\hline$(2,1,1,2)$ & $q+127$ \\
\hline$(1,1,1,1,2)$ & $q+185$ \\
\hline$(1,1,1,2,1)$ & 173 \\
\hline$(1,1,2,1,1)$ & $q+170$ \\
\hline$(1,1,1,1,1,1)$ & $q+273$ \\
\hline
\end{tabular}

\section{Appendix Ranks of partition matrices Anton Evseev and George Wellen}

Let $S$ be a subset of $\mathbb{N}$. If $k \in \mathbb{Z}$, let $p(k, S)$ be the number of partitions $\lambda$ such that $|\lambda|=k$ and $\lambda_{i} \in S$ for all $i$. Recall that $r(k, S)$ is the number of partitions $\lambda$ such that $\lambda_{i} \in S$ for all $i$ and, for each $s \in S$, there exists $i$ such that $\lambda_{i}=s$.

Fix a positive integer $m$, and let $n=m(m+1) / 2$. Let $\mathrm{P}$ be the matrix whose columns are indexed by non-empty subsets of $[1, m]$, whose rows are indexed by nonnegative integers and whose $(k, S)$ entry is $p(k, S)$. Let R be the matrix whose rows are indexed by positive integers $k$ and whose columns are indexed by non-empty subsets $S$ of $[1, m]$ with the $(k, S)$ entry equal to $r(k, S)$. Thus, $\mathrm{P}$ and $\mathrm{R}$ have infinitely many rows and $2^{m}-1$ columns each. Our aim is to find out the ranks of $\mathrm{P}$ and $\mathrm{R}$ and to find linear relations between rows of $\mathrm{P}$ and $\mathrm{R}$.

If $S \subseteq[1, m]$, let

$$
P_{S}(X)=\sum_{k=0}^{\infty} p(k, S) X^{k}
$$

be the generating function of the sequence $(p(k, S))_{s=0}^{\infty}$. Here, and in the sequel, $X$ is a formal variable, and all expressions involving $X$ are assumed to be elements of the ring $\mathbb{Q}[[X]]$ of formal power series over $\mathbb{Q}$.

Observe that

$$
P_{\{i\}}(X)=\sum_{k \geq 0} X^{i k}=\frac{1}{1-X^{i}} .
$$

It follows that, for any non-empty finite subset $S \subset \mathbb{N}$,

$$
P_{S}(X)=\frac{1}{\prod_{i \in S}\left(1-X^{i}\right)}
$$

Also, by the inclusion-exclusion formula, for all $S \subseteq[1, m]$ and all $k \in \mathbb{N}$,

$$
r(k, S)=\sum_{S^{\prime} \subseteq S, S \neq \varnothing}(-1)^{|S|-\left|S^{\prime}\right|} p(k, S) .
$$

(Note that $p(k, \varnothing)=0$ for $k>0$.) The following result can be easily proved by induction. 
Lemma 1. For every natural number $k \in[1, n]$ there exists a non-empty subset $S_{k}$ of $[1, m]$ such that $k=\sum_{i \in S_{k}} i$.

Let

$$
\Delta(X)=\prod_{i=1}^{m}\left(1-X^{i}\right) .
$$

Define integers $c_{0}, c_{1}, \ldots, c_{n}$ by

$$
\Delta(X)=\sum_{i=0}^{n} c_{i}^{(m)} X^{i}
$$

Theorem 2. $\operatorname{rank}(\mathrm{P})=n=m(m+1) / 2$. Moreover, for all $k \geq n$ and all non-empty $S \subseteq[1, m]$,

$$
p(k, S)=-\sum_{j=k-n}^{k-1} c_{k-j} p(j, S) .
$$

Proof. Let $S$ be a non-empty subset of $[1, m]$. By (24),

$$
\begin{aligned}
P_{S}(X) \Delta(X) & =\prod_{i \in[1, m] \backslash S}\left(1-X^{i}\right), \text { hence, } \\
\sum_{k=0}^{\infty} \sum_{j=\max (0, k-n)}^{k} c_{k-j} p(j, S) X^{k} & =\prod_{i \in[1, m] \backslash S}\left(1-X^{i}\right) .
\end{aligned}
$$

If $k \geq n$, the coefficient in $X^{k}$ on the right-hand side is 0 (since $S \neq \varnothing$ ), so

$$
\sum_{j=k-n}^{k} c_{k-j} p(j, S)=0 .
$$

Then the expression for $p(k, S)$ follows from the fact that $c_{0}=1$. It follows that $\operatorname{rank}(\mathrm{P}) \leq n$.

To show that $\operatorname{rank}(\mathrm{P}) \geq n$, it is enough to prove that the polynomials $P_{S}(X) \Delta(X)$ span a subspace of dimension at least $n$ in $\mathbb{Q}[[X]]$ as $S$ varies among the non-empty subsets of $[1, m]$. By (26),

$$
\operatorname{deg}\left(P_{S} \cdot \Delta\right)=\sum_{i \in[1, m] \backslash S} i=n-\sum_{i \in S} i
$$

Hence, by Lemma 1 for each integer $k \in[0, n-1]$, there exists a non-empty $S \subseteq[1, m]$ such that $\operatorname{deg}\left(P_{S} \cdot \Delta\right)=k$. The result follows.

Corollary 3. $\operatorname{rank}(\mathrm{R})=n$. Moreover, for all $k>n$ and all non-empty $S \subseteq$ $[1, m]$,

$$
r(k, S)=-\sum_{j=k-n}^{k-1} c_{k-j} r(j, S) .
$$

Proof. The expression for $r(k, S)$ follows from Theorem 2 and (25). By (25), $\operatorname{rank}(\mathrm{R})$ is equal to the rank of the matrix obtained by removing the 0 -row from P. By Theorem 2,

$$
c_{n} p(0, S)=-\sum_{j=1}^{n} c_{n-j} p(j, S),
$$

so the 0 -row of $\mathrm{P}$ is a linear combination of the next $n$ rows $\left(c_{n} \neq 0\right)$. Hence, $\operatorname{rank}(\mathrm{R})=n$. 
Define $d_{j}=\sum_{i=0}^{n-j} c_{i}$ for $j=1,2, \ldots n$.

Proposition 4. For all non-empty $S \subseteq[1, m]$,

$$
\sum_{j=1}^{n} d_{j} r(j, S)=\sum_{j=1}^{n} d_{j} p(j, S)= \begin{cases}1 & \text { if } S=[1, m] \\ 0 & \text { otherwise }\end{cases}
$$

Proof. Let $f(X)=\left(P_{S}(X)-1\right) \Delta(X)$. Then

$$
f(X)=\prod_{i \in[1, m] \backslash S}\left(1-X^{i}\right)-\Delta(X) .
$$

Thus, $f(X)$ is a polynomial of degree $n$, and $f(1)$ is the sum of the coefficients of $f(X)$ in $X^{0}, X^{1}, \ldots, X^{n}$. On the other hand,

$$
f(X)=\sum_{j=1}^{\infty} \sum_{i=0}^{n} c_{i} p(j, S) X^{i+j} .
$$

Therefore,

$$
f(1)=\sum_{j=1}^{n} \sum_{i=0}^{n-j} c_{i} p(j, S)=\sum_{j=1}^{n} d_{j} p(j, S) .
$$

Hence,

$$
\sum_{j=1}^{n} d_{j} p(j, S)=f(1)= \begin{cases}1 & \text { if } S=[1, m] \\ 0 & \text { otherwise. }\end{cases}
$$

By (25), $\sum_{j=1}^{n} d_{j} r(j, S)$ is equal to this too.

\section{References}

[1] M. Auslander, I. Reiten, and S.O. Smalø, Representation theory of Artin algebras, Cambridge University Press, 1994.

[2] N. Bourbaki, Algebre commutative. Chapitre VI, Hermann, Paris, 1964.

[3] C.W. Curtis and I. Reiner, Methods of representation theory: with applications to finite groups and orders, vol. 1, John Wiley \& Sons, Inc., 1981.

[4] A. Evseev, Groups: uniformity questions and zeta functions, D.Phil. thesis, Oxford, 2007.

[5] P. Magyar, J. Weyman, and A. Zelevinsky, Multiple flag varieties of finite type, Adv. Math. 141 (1999), no. 1, 97-118.

[6] S.H. Murray, Representations of parabolic and Borel subgroups, to appear in Comm. Algebra.

[7] Conjugacy classes in maximal parabolic subgroups of general linear groups, J. Algebra 233 (2000), 135-155.

[8] A. Vera-López and J.M. Arregi, Conjugacy classes in Sylow p-subgroups of $\mathrm{Gl}(n, q)$, J. Algebra 152 (1992), no. 1, 1-19.

[9] $\_$Some algorithms for the calculation of conjugacy classes in the Sylow p-subgroups of $\mathrm{Gl}(n, q)$, J. Algebra 177 (1995), 899-925.

[10] Conjugacy classes in unitriangular matrices, Linear Algebra Appl. 370 (2003), 85-124. 